\title{
Sharp Estimates for the Ornstein-Uhlenbeck Operator
}

\author{
GIANCARLO MAUCERI - STEFANO MEDA - PETER SJÖGREN
}

\begin{abstract}
Let $\mathcal{L}$ be the Ornstein-Uhlenbeck operator which is self-adjoint with respect to the Gauss measure $\gamma$ on $\mathbb{R}^{d}$. We prove a sharp estimate of the operator norm of the imaginary powers of $\mathcal{L}$ on $L^{p}(\gamma), 1<p<\infty$. Then we use this estimate to prove that if $b$ is in $[0, \infty)$ and $M$ is a bounded holomorphic function in the sector $\{z \in \mathbb{C}:|\arg (z-b)|<\arcsin |2 / p-1|\}$ and satisfies a Hörmanderlike condition of (nonintegral) order greater than one on the boundary, then the operator $M(\mathcal{L})$ is bounded on $L^{p}(\gamma)$. This improves earlier results of the authors with J. García-Cuerva and J.L. Torrea.
\end{abstract}

Mathematics Subject Classification (2000): 47A60 (primary); 47D03, 60G15 (secondary).

\section{0. - Introduction}

The purpose of this paper is to obtain a sharp functional calculus for the Ornstein-Uhlenbeck operator acting on the $L^{p}$ spaces with respect to the Gauss measure on $\mathbb{R}^{d}$, i.e., the probability measure $\gamma$ defined by

$$
\mathrm{d} \gamma(x)=\pi^{-d / 2} \exp \left(-|x|^{2}\right) \mathrm{d} \lambda(x)
$$

where $\lambda$ denotes Lebesgue measure on $\mathbb{R}^{d}$. The Ornstein-Uhlenbeck operator

$$
-\frac{1}{2} \Delta+x \cdot \nabla
$$

is essentially self-adjoint on $L^{2}(\gamma)$. Its closure $\mathcal{L}$ has spectral resolution

$$
\mathcal{L} f=\sum_{n=0}^{\infty} n \mathcal{P}_{n} f \quad \forall f \in \operatorname{Dom}(\mathcal{L}),
$$

Work partially supported by the European Commission Network "Harmonic Analysis and Related Problems", the Progetto Cofinanziato MIUR "Analisi Armonica" and the Gruppo Nazionale INdAM per l'Analisi Matematica, la Probabilità e le loro Applicazioni

Pervenuto alla Redazione il 7 gennaio 2004. 
where $\mathcal{P}_{n}$ is the orthogonal projection onto the linear span of Hermite polynomials of degree $n$ in $d$ variables. Suppose that $M: \mathbb{N} \rightarrow \mathbb{C}$ is a bounded sequence. The operator $M(\mathcal{L})$, defined by

$$
M(\mathcal{L}) f=\sum_{n=0}^{\infty} M(n) \mathcal{P}_{n} f \quad \forall f \in L^{2}(\gamma),
$$

is bounded on $L^{2}(\gamma)$ by the spectral theorem. We call $M(\mathcal{L})$ the spectral operator associated to the spectral multiplier $M$. If $M(\mathcal{L})$ extends to a bounded operator on $L^{p}(\gamma)$ for some $p$ in $[1, \infty)$, we say that $M$ is an $L^{p}(\gamma)$ spectral multiplier.

The operator $\mathcal{L}$ generates a markovian semigroup. It is well known that the existence of an $H^{\infty}$ functional calculus in $L^{p}$ for such generators is related to the growth of the $L^{p}$ operator norm of their imaginary powers (see, for instance, [1], [2] and the references there, [7] and [11]). However, the imaginary powers of our operator $\mathcal{L}$ are not well defined since 0 is an eigenvalue. For $\mathcal{L}$, or more generally for any selfadjoint operator $\mathcal{G}$, we shall replace the imaginary powers by the operators $\mathcal{G}_{+}^{i u}$, defined by

$$
\mathcal{G}_{+}^{i u}=\int_{0^{+}}^{\infty} \zeta^{i u} \mathrm{~d} \mathcal{E}_{\zeta} \quad \forall u \in \mathbb{R},
$$

where $\left\{\mathcal{E}_{\zeta}: \zeta \in \mathbb{R}\right\}$ is the spectral resolution of $\mathcal{G}$.

On the one hand, the lower bound

$$
\left\|\mathcal{L}_{+}^{i u}\right\|_{L^{p}(\gamma)} \geq e^{\phi_{p}^{*}|u|} \quad \forall u \in \mathbb{R},
$$

where $\phi_{p}^{*}=\arcsin |2 / p-1|$ and $1<p<\infty$, is implicit in [7]. This estimate has been reproved and extended to more general operators in a recent paper by W. Hebisch, Mauceri and Meda [8].

On the other hand, it is known [7, formula (2), p. 417] that there exists a constant $C$ such that

$$
\left\|\mathcal{L}_{+}^{i u}\right\|_{L^{p}(\gamma)} \leq C(1+|u|)^{5 / 2} e^{\phi_{p}^{*}|u|} \quad \forall u \in \mathbb{R} .
$$

One of the main results of this paper shows that the factor $(1+|u|)^{5 / 2}$ can be deleted here, so that the lower bound given above is sharp; see Theorem 4.3 below.

As a consequence, we may improve the $H^{\infty}$ functional calculus for $\mathcal{L}$ in $L^{p}(\gamma)$ proved in [7, Theorem 1 (i)], by reducing the degree of smoothness of the multiplier needed on the boundary of the critical sector $\mathbf{S}_{\phi_{p}^{*}}=\{z \in \mathbb{C}$ : $\left.\arg z \in\left(-\phi_{p}^{*}, \phi_{p}^{*}\right)\right\}$. The precise statement of our result (Theorem 1.2 below, in particular the case $b=0)$ involves the Banach spaces $H^{\infty}\left(\mathbf{S}_{\phi_{p}^{*}} ; J\right)$, defined in Section 3, of all bounded holomorphic functions in the sector $\mathbf{S}_{\phi_{p}^{*}}$ satisfying a Hörmander-like condition of order $J$ on the boundary. It may be worth 
noticing that Theorem 1.2 requires holomorphy in a sector whose aperture $\phi_{p}^{*}$ is smaller than that prescribed in M. Cowling's result [1] for generators of general submarkovian semigroups.

Now, observe that for every $p$ in $(1, \infty)$ the $L^{p}(\gamma)$ spectrum of $\mathcal{L}$ is $\mathbb{N}$ [4, p. 115]. Thus the spectral projections $\mathcal{P}_{n}$ are bounded, though not uniformly, in $L^{p}(\gamma)$. Therefore it is natural to speculate whether holomorphy in the whole sector $\mathbf{S}_{\phi_{p}^{*}}$ is really needed for $L^{p}(\gamma)$ spectral multipliers of $\mathcal{L}$. An application of the Dunford functional calculus shows that if $M: \mathbb{N} \rightarrow \mathbb{C}$ is a bounded sequence, which is the restriction to $\mathbb{N}$ of a function holomorphic in a neighbourhood of infinity, then $M(\mathcal{L})$ extends to a bounded operator on $L^{p}(\gamma)$ for all $p$ in $(1, \infty)$. Thus, it seems reasonable to conjecture that a bounded sequence $M$, which is the restriction to $\mathbb{N}$ of a function holomorphic and bounded on the intersection of $\mathbf{S}_{\phi_{p}^{*}}$ and a neighbourhood of infinity, and which satisfies a Hörmander type condition on the part at infinity of the boundary of $\mathbf{S}_{\phi_{p}^{*}}$ is an $L^{p}(\gamma)$ spectral multiplier of $\mathcal{L}$.

In fact, a stronger result holds. Our main result, Theorem 1.2 below, states that if $b$ is nonnegative and $M$ is a bounded sequence which on $\mathbb{N} \cap(b, \infty)$ coincides with the restriction of a function which is holomorphic and bounded on the translated sector $b+\mathbf{S}_{\phi_{p}^{*}}$ and satisfies Hörmander-like conditions of suitable order on the boundary of $b+\mathbf{S}_{\phi_{p}^{*}}$, then $M$ is an $L^{p}(\gamma)$ spectral multiplier of $\mathcal{L}$. The statement of Theorem 1.2 is contained in Section 1, where we also show how to reduce its proof to the problem of establishing for each nonnegative $b$ a suitable bounded holomorphic functional calculus in $L^{p}(\gamma)$ for the operator

$$
\mathcal{L}_{b}=\mathcal{L}-b \mathcal{I}
$$

Since $\mathcal{L}_{b}$ does not generate a symmetric contraction semigroup, the required functional calculus for $\mathcal{L}_{b}$ is not a direct consequence of the results in [11], [3] and [7, Section 2]. To overcome this difficulty, we proceed as follows.

First, in Section 2, we consider a self-adjoint operator $\mathcal{G}$ acting on $L^{2}(X)$, where $X$ is a $\sigma$-finite measure space, and we prove that if $\mathcal{G}$ has a functional calculus of Laplace transform type in $L^{p}(X)$, a control on the growth of $\left\|\mathcal{G}_{+}^{i u}\right\|_{p}$ as $|u|$ tends to infinity implies that $\mathcal{G}$ admits a bounded functional calculus in $L^{p}(X)$ on suitable sectors. The proof combines results of Meda [11, Theorem 1], Cowling and Meda [3, Theorem 2.1] and [7, Theorem 2.2].

Then, in Section 3, we prove that for every $p$ in $(1, \infty) \backslash\{2\}$ the operator $\mathcal{L}_{b}$ admits a functional calculus of Laplace transform type in $L^{p}(\gamma)$ (see Theorem 3.2 below).

Finally, in Theorem 4.3 we show that for every nonnegative $b$ there exists a constant $C$ such that

$$
\left\|\left(\mathcal{L}_{b}\right)_{+}^{i u}\right\|_{L^{p}(\gamma)} \leq C e^{\phi_{p}^{*}|u|} \quad \forall u \in \mathbb{R} .
$$

The proof of Theorem 4.3 follows closely the strategy of that of [7, Proposition 3.1 and Proposition 3.2], and it is contained in Section 4, except for the proof of claim (4.11), which is rather technical and is deferred to Section 5. 
As a consequence of the analysis performed in Sections 3, 4 and 5, we obtain the bounded holomorphic functional calculus in $L^{p}(\gamma)$ for the operators $\mathcal{L}_{b}, b \geq 0$, required to conclude the proof of Theorem 1.2.

\section{1. - The multiplier result for $\mathcal{L}$}

Suppose that $J$ is a positive number and that $\psi$ is in $(0, \pi / 2)$. Let $\mathbf{S}_{\psi}$ denote the open sector

$$
\{z \in \mathbb{C}: \arg z \in(-\psi, \psi)\}
$$

and let $\varphi: \mathbb{R} \rightarrow[0,1]$ be a smooth function, supported in $(1 / 4,4)$ and equal to one on $[1 / 2,2]$. We denote by $H^{J}(\mathbb{R})$ the standard Sobolev space on $\mathbb{R}$, modelled over $L^{2}(\mathbb{R})$, and by $H^{\infty}\left(\mathbf{S}_{\psi} ; J\right)$ the Banach space of all $M$ in $H^{\infty}\left(\mathbf{S}_{\psi}\right)$ for which there exists a constant $C$ such that

$$
\sup _{t>0}\left\|\varphi M\left(e^{i \varepsilon \psi} t \cdot\right)\right\|_{H^{J}(\mathbb{R})} \leq C, \quad \varepsilon \in\{-1,1\}
$$

endowed with the norm

$$
\|M\|_{\psi ; J}=\inf \{C:(1.1) \text { holds }\}
$$

Condition (1.1) is called a Hörmander condition of order $J$ [9]. Note that (1.1) implies that $\sup _{z \in \mathbf{S}_{\psi}}|M(z)| \leq 2 C$, if $J>1 / 2$.

If $b$ is in $\mathbb{R}^{+}$and $\theta$ is in $(0, \pi / 2)$, let $b+\mathbf{S}_{\theta}$ denote the translated sector

$$
\{z \in \mathbb{C}: \arg (z-b) \in(-\theta, \theta)\}
$$

Suppose that $f:(b, \infty) \rightarrow \mathbb{C}$. We shall denote by ${ }_{b} f$ the function defined by

$$
{ }_{b} f(x)=f(b+x) \quad \forall x \in \mathbb{R}^{+} .
$$

We denote by $H^{\infty}\left(b+\mathbf{S}_{\psi} ; J\right)$ the Banach space of all $M$ in $H^{\infty}\left(b+\mathbf{S}_{\psi}\right)$ such that ${ }_{b} M$ is in $H^{\infty}\left(\mathbf{S}_{\psi} ; J\right)$, with norm

$$
\|M\|_{\psi, b ; J}=\left\|_{b} M\right\|_{\psi ; J}
$$

We note that requiring that a function $M$ is in $H^{\infty}\left(b+\mathbf{S}_{\psi} ; J\right)$, for some $b>0$, is weaker than assuming that $M \in H^{\infty}\left(\mathbf{S}_{\psi} ; J\right)$. Indeed, one has the following

Proposition 1.1. For every $J, b>0$ the space $H^{\infty}\left(\mathbf{S}_{\psi} ; J\right)$ embeds continuously in $H^{\infty}\left(b+\mathbf{S}_{\psi} ; J\right)$. 
Proof. Let $J, b>0$. Given $M$ in $H^{\infty}\left(\mathbf{S}_{\psi} ; J\right)$ we shall prove that $M$ is also in $H^{\infty}\left(b+\mathbf{S}_{\psi} ; J\right)$, with a corresponding norm estimate. We must estimate the $H^{J}(\mathbb{R})$ norm of the functions $s \mapsto \varphi(s) M\left(b+e^{ \pm i \psi} t s\right)$, uniformly in $t>0$. Notice that these functions are supported in $(1 / 4,4)$.

We choose the plus sign in the exponent and fix $t>0$. Let $\Gamma$ be the contour defined by the boundary of the triangle $\mathbf{S}_{\psi} \cap\{\operatorname{Re} z<10 b+10 t\}$, taken in the positive sense. For $z$ in the segment $I_{t}=\left\{b+e^{i \psi} t s: 1 / 4<s<4\right\}$ clearly

$$
M(z)=\frac{1}{2 \pi i} \int_{\Gamma} \frac{M(z)}{\zeta-z} \mathrm{~d} \zeta
$$

Observe that for $z \in I_{t}$ and $\zeta \in \Gamma$, one has $|\zeta-z|>c b$, for some $c>0$, and that the length of $\Gamma$ is at most $C b+C t$.

In the case $t \leq b$, we differentiate the above Cauchy formula and get

$$
\left|M^{(\ell)}(z)\right| \leq C b^{-\ell}\|M\|_{\infty} \leq C t^{-\ell}\|M\|_{\infty},
$$

for $z \in I_{t}$ and $\ell \geq 0$. This immediately implies the required estimate.

Assume now $t>b$. We introduce a function $\eta$ in $C_{c}^{\infty}(\mathbb{R})$ supported in $[1 / 10,10]$ and with $\eta(r)=1$ for $1 / 8 \leq r \leq 8$. Define $\tilde{\eta}$ on $\Gamma$ by letting $\tilde{\eta}\left(e^{i \psi} t r\right)=\eta(r)$ when $e^{i \psi} t r \in \Gamma$ and letting $\tilde{\eta}=0$ on the other two sides of the triangle.

Write

$$
\begin{aligned}
\int_{\Gamma} \frac{M(\zeta)}{\zeta-z} \mathrm{~d} \zeta & =\int_{\Gamma} \frac{(1-\tilde{\eta}(\zeta)) M(\zeta)}{\zeta-z} \mathrm{~d} \zeta+\int_{\Gamma} \frac{\tilde{\eta}(\zeta) M(\zeta)}{\zeta-z} \mathrm{~d} \zeta \\
& =M_{1}(z)+M_{2}(z) .
\end{aligned}
$$

For $z$ in $I_{t}$ and $\zeta$ in $\Gamma$ with $\tilde{\eta}(\zeta) \neq 1$, it is easy to see that $|\zeta-z| \geq c t$ for some $c>0$. To deal with the derivatives of $M_{1}$, we can therefore differentiate under the integral sign and get

$$
\left|M_{1}^{(\ell)}(z)\right| \leq C t^{-\ell}\|M\|_{\infty}, \quad \forall z \in I_{t},
$$

which implies the required estimate as before.

In $M_{2}$ we make the change of variables $\zeta=e^{i \psi} t r$, getting

$$
\begin{aligned}
M_{2}\left(b+e^{i \psi} t s\right) & =-\int \frac{\eta(r) M\left(e^{i \psi} t r\right)}{r-e^{-i \psi} t^{-1} b-s} \mathrm{~d} r \\
& =\int \frac{\eta(r) M\left(e^{i \psi} t r\right)}{s+b t^{-1} \cos \psi-r-i b t^{-1} \sin \psi} \mathrm{d} r .
\end{aligned}
$$

This is the convolution in $\mathbb{R}$, taken at $s+b t^{-1} \cos \psi$, of the two functions $g_{t}(r)=\eta(r) M\left(e^{i \psi} t r\right)$ and $K_{t}(r)=\left(r-i b t^{-1} \sin \psi\right)^{-1}$. The assumption $M \in$ $H^{\infty}\left(\mathbf{S}_{\psi} ; J\right)$ implies that $g_{t} \in H^{J}(\mathbb{R})$, uniformly in $t$. As seen via Fourier transform, convolution by $K_{t}$ defines a bounded operator on $L^{2}(\mathbb{R})$, uniformly in $t$. The same boundedness then also holds on the Sobolev spaces $H^{\ell}(\mathbb{R})$, for integer and fractional $\ell$. Since multiplication by $\varphi$ causes no problem, the required estimate follows. The argument for $s \mapsto \varphi(s) M\left(b+e^{-i \psi} t s\right)$ is similar. 
For every nonnegative number $b$ let $\mathcal{L}_{b}$ denote the self-adjoint operator $\mathcal{L}-b \mathcal{I}$. The main result of this paper is the following.

THeOREM 1.2. Suppose that $p$ is in $(1, \infty) \backslash\{2\}$, that $b$ is in $[0, \infty)$, and that $J$ is in $(1, \infty)$. Let $M: \mathbb{N} \rightarrow \mathbb{C}$ be a bounded sequence and assume that there exists a function $\widetilde{M}$ in $H^{\infty}\left(b+\mathbf{S}_{\phi_{p}^{*}} ; J\right)$ such that

$$
\widetilde{M}(k)=M(k) \quad \forall k \in \mathbb{N} \cap(b, \infty) .
$$

Then $M(\mathcal{L})$ extends to a bounded operator on $L^{p}(\gamma)$ and there exists a constant $C$ such that

$$
\|M(\mathcal{L})\|_{p} \leq C\left(\|M\|_{\infty}+\|\widetilde{M}\|_{\phi_{p}^{*}, b ; J}\right)
$$

for all $M$ as above.

STRUCTURE OF THE PROOF. We may extend $\widetilde{M}$ to a function on $(-\infty, b] \cup$ $\left(b+\mathbf{S}_{\phi_{p}^{*}}\right)$, which vanishes on $(-\infty, b]$. We abuse the notation and denote by $\widetilde{M}$ this extension. Clearly

$$
M(\mathcal{L})=\sum_{j<b} M(j) \mathcal{P}_{j}+\widetilde{M}(\mathcal{L})
$$

Note that by spectral theory

$$
\begin{aligned}
\widetilde{M}(\mathcal{L}) & =\sum_{j>b}{ }_{b} \widetilde{M}(j-b) \mathcal{P}_{j} \\
& ={ }_{b} \widetilde{M}\left(\mathcal{L}_{b}\right) .
\end{aligned}
$$

Then

$$
\begin{aligned}
\|M(\mathcal{L})\|_{p} & \leq \sum_{j \leq b}|M(j)|\left\|\mathcal{P}_{j}\right\|_{p}+\left\|_{b} \widetilde{M}\left(\mathcal{L}_{b}\right)\right\|_{p} \\
& \leq C\|M\|_{\infty}+\|\|_{b} \widetilde{M}\left(\mathcal{L}_{b}\right) \|_{p} .
\end{aligned}
$$

Note that ${ }_{b} \widetilde{M}$ vanishes on $(-\infty, 0]$ and its restriction to $\mathbb{R}^{+}$is in $H^{\infty}\left(\mathbf{S}_{\phi_{p}^{*}} ; J\right)$. Thus, to conclude the proof of the theorem, it suffices to show that operators of the form $M\left(\mathcal{L}_{b}\right)$, where $M: \mathbb{R} \rightarrow \mathbb{C}$ vanishes on $(-\infty, 0]$ and is the restriction to $\mathbb{R}^{+}$of a function in $H^{\infty}\left(\mathbf{S}_{\phi_{p}^{*}} ; J\right)$ for some $J$ in $(1, \infty)$, are bounded on $L^{p}(\gamma)$. As we announced in the introduction, the proof of this result will follow from the analysis carried out in the next sections. We summarize the main steps of the argument.

By Theorem 3.2, the operator $\mathcal{L}_{b}$ admits a functional calculus of Laplace transform type in $L^{p}(\gamma)$ (see the beginning of Section 2 for the definition). Furthermore, by Theorem 4.3 below there exists a constant $C$ such that

$$
\left\|\left(\mathcal{L}_{b}\right)_{+}^{i u}\right\|_{L^{p}(\gamma)} \leq C e^{\phi_{p}^{*}|u|} \quad \forall u \in \mathbb{R} .
$$


Thus, $\mathcal{L}_{b}$ satisfies the hypotheses of Theorem 2.1 (ii) (with $\phi_{p}^{*}$ in place of $\theta$ ), so there exists a constant $C$ such that

$$
\begin{aligned}
\left\|_{b} \widetilde{M}\left(\mathcal{L}_{b}\right)\right\|_{p} & \leq C\left\|_{b} \widetilde{M}\right\|_{\phi_{p}^{*} ; J} \\
& =C\|\widetilde{M}\|_{\phi_{p}^{*}, b ; J} .
\end{aligned}
$$

The proof of the theorem is complete, except for the auxiliary results established in the following sections.

\section{2. - An abstract result}

We say that a function $M: \mathbb{R}^{+} \rightarrow \mathbb{C}$ is of Laplace transform type if there exists a function $m$ in $L^{\infty}\left(\mathbb{R}^{+}\right)$such that

$$
M(\lambda)=\lambda \int_{0}^{\infty} m(s) e^{-\lambda s} \mathrm{~d} s \quad \forall \lambda \in \mathbb{R}^{+} .
$$

Note that $M$ then extends to a bounded holomorphic function in the sector $\mathbf{S}_{\theta}$ for all $\theta$ in $(0, \pi / 2)$. In particular, $M$ is bounded on $\mathbb{R}^{+}$.

In this section we consider a (possibly unbounded) self-adjoint operator $\mathcal{G}$ on $L^{2}(X)$, where $X$ is a $\sigma$-finite measure space. Let $\left\{\mathcal{E}_{\zeta}\right\}_{\zeta \in \mathbb{R}}$ denote the spectral resolution of the identity for which

$$
\mathcal{G} f=\int_{-\infty}^{\infty} \zeta \mathrm{d} \mathcal{E}_{\zeta} f \quad \forall f \in \operatorname{Dom}(\mathcal{G}) .
$$

If $p$ is in $(1, \infty) \backslash\{2\}$, we say that $\mathcal{G}$ admits a functional calculus of Laplace transform type in $L^{p}(X)$ if there exists a constant $C$ such that for every $M$ : $\mathbb{R} \rightarrow \mathbb{C}$, which vanishes on $(-\infty, 0]$ and whose restriction to $\mathbb{R}^{+}$is of Laplace transform type (as in (2.1)), the operator $M(\mathcal{G})$, initially defined on $L^{2}(X) \cap$ $L^{p}(X)$, extends to a bounded operator on $L^{p}(X)$, and

$$
\|M(\mathcal{G})\|_{L^{p}(X)} \leq C\|m\|_{\infty} .
$$

E.M. Stein [13] proved that infinitesimal generators of diffusion semigroups admit a functional calculus of Laplace transform type in $L^{p}$ for all $p$ in $(1, \infty)$. This result was subsequently extended to generators of symmetric contraction semigroups by M. Cowling [1]. In Section 3, we shall show that $\mathcal{L}_{b}$ admits a functional calculus of Laplace transform type, although it does not generate a symmetric contraction semigroup.

For the statement of the next result, we need the following notation. Recall that the Mellin transform $\mathcal{M} f$ of a function $f$ in $L^{1}\left(\mathbb{R}^{+}, \mathrm{d} \zeta / \zeta\right)$ is defined by

$$
\mathcal{M} f(u)=\int_{0}^{\infty} f(\zeta) \zeta^{-i u} \frac{\mathrm{d} \zeta}{\zeta} \quad \forall u \in \mathbb{R}
$$


Let $M$ be a complex-valued function, bounded and Borel measurable on $\mathbb{R}^{+}$. Given a positive integer $N$, we denote by $M_{N}: \mathbb{R}^{+} \times \mathbb{R}^{+} \rightarrow \mathbb{C}$ the function defined by

$$
M_{N}(t, \zeta)=(t \zeta)^{N} e^{-t \zeta} M(\zeta),
$$

and by $\mathcal{M} M_{N}(t, \cdot)$ the Mellin transform of $M_{N}(t, \cdot)$.

THEOREM 2.1. Suppose that $p$ is in $(1, \infty) \backslash\{2\}$, and that $\mathcal{G}$ is a self-adjoint operator on $L^{2}(X)$ which admits a functional calculus of Laplace transform type in $L^{p}(X)$.

(i) For each positive integer $N$ there exists a constant $C$ such that for every bounded Borel measurable function $M: \mathbb{R} \rightarrow \mathbb{C}$ which vanishes on $(-\infty, 0]$

$$
\|M(\mathcal{G})\|_{p} \leq C \int_{-\infty}^{\infty} \sup _{t>0}\left|\mathcal{M} M_{N}(t, u)\right|\left\|\mathcal{G}_{+}^{i u}\right\|_{p} \mathrm{~d} u .
$$

(ii) Suppose further that $\theta$ is in $(0, \pi / 2)$, and that there exists a constant $C$ such that

$$
\left\|\mathcal{G}_{+}^{i u}\right\|_{p} \leq C e^{\theta|u|} \quad \forall u \in \mathbb{R} .
$$

Then for each $J$ in $(1, \infty)$ there exists a constant $C$ such that for every $M$ : $(-\infty, 0] \cup \mathbf{S}_{\theta} \rightarrow \mathbb{C}$ which vanishes on $(-\infty, 0]$ and whose restriction to $\mathbf{S}_{\theta}$ is in $H^{\infty}\left(\mathbf{S}_{\theta} ; J\right)$

$$
\|M(\mathcal{G})\|_{p} \leq C\|M\|_{\theta ; J} .
$$

PROOF. The proof of (i) is very similar to the proof of [3, Theorem 2.1]. There the following formula was proved:

$$
M(\zeta)=\frac{1}{2 \pi \Gamma(N+1)} \int_{-\infty}^{\infty} A_{u}(\zeta) c(u) \zeta^{i u} \mathrm{~d} u \quad \forall \zeta \in \mathbb{R}^{+},
$$

where

$$
c(u)=\sup _{t>0}\left|\mathcal{M} M_{N}(t, u)\right|, \quad a_{u}(t)=\frac{\mathcal{M} M_{N}(t, u)}{c(u)} \quad \forall u \in \mathbb{R} \quad \forall t \in \mathbb{R}^{+},
$$

and

$$
A_{u}(\zeta)=\int_{0}^{\infty} a_{u}(t) t \zeta e^{-t \zeta / 2} \frac{\mathrm{d} t}{t} \quad \forall \zeta \in \mathbb{R}^{+} .
$$

We extend $A_{u}$ to $\mathbb{R}$, by requiring that the extended function vanishes on $(-\infty, 0]$. Here we abuse the notation and denote this extension also by $A_{u}$. Since $\mathcal{G}$ admits a functional calculus of Laplace transform type in $L^{p}(X)$, the operator $A_{u}(\mathcal{G})$ is bounded on $L^{p}(X)$. Then (2.2) implies that

$$
\begin{aligned}
\left\|A_{u}(\mathcal{G})\right\|_{p} & \leq C\left\|a_{u}\right\|_{\infty} \\
& \leq C .
\end{aligned}
$$


Thus,

$$
\int_{-\infty}^{\infty}\left\|A_{u}(\mathcal{G})\right\|_{p} c(u)\left\|\mathcal{G}_{+}^{i u}\right\|_{p} \mathrm{~d} u \leq C \int_{-\infty}^{\infty} c(u)\left\|\mathcal{G}_{+}^{i u}\right\|_{p} \mathrm{~d} u,
$$

which is finite by assumption. Since $M$ vanishes on $(-\infty, 0]$, at least formally,

$$
\begin{aligned}
M(\mathcal{G}) & =\int_{0+}^{\infty} M(\zeta) \mathrm{d} \mathcal{E}_{\zeta} \\
(\operatorname{by}(2.3)) & =\frac{1}{2 \pi \Gamma(N+1)} \int_{0+}^{\infty} \int_{-\infty}^{\infty} A_{u}(\zeta) c(u) \zeta^{i u} \mathrm{~d} u \mathrm{~d} \mathcal{E}_{\zeta} \\
& =\frac{1}{2 \pi \Gamma(N+1)} \int_{-\infty}^{\infty} A_{u}(\mathcal{G}) c(u) \mathcal{G}_{+}^{i u} \mathrm{~d} u
\end{aligned}
$$

It is not hard to justify the last equality. Indeed,

$$
\begin{aligned}
\int_{-\infty}^{\infty} \int_{0+}^{\infty} c(u)\left|A_{u}(\zeta) \zeta^{i u}\right|\left|\mathrm{d}\left\langle\mathcal{E}_{\zeta} f, g\right\rangle\right| \mathrm{d} u & \leq 2\left\|a_{u}\right\|_{\infty} \int_{0+}^{\infty}\left|\mathrm{d}\left\langle\mathcal{E}_{\zeta} f, g\right\rangle\right| \int_{-\infty}^{\infty} c(u) \mathrm{d} u \\
& \leq 2\left\|a_{u}\right\|_{\infty}\|f\|_{2}\|g\|_{2} \int_{-\infty}^{\infty} c(u)\left\|\mathcal{G}_{+}^{i u}\right\|_{p} \mathrm{~d} u \\
& <\infty
\end{aligned}
$$

Note that the second inequality here follows from the elementary fact that $1=\left\|\mathcal{G}_{+}^{i u}\right\|_{2} \leq\left\|\mathcal{G}_{+}^{i u}\right\|_{p}$. Thus,

$$
\begin{aligned}
\|M(\mathcal{G})\|_{p} & =\frac{1}{2 \pi \Gamma(N+1)}\left\|\int_{-\infty}^{\infty} A_{u}(\mathcal{G}) c(u) \mathcal{G}_{+}^{i u} \mathrm{~d} u\right\|_{p} \\
& \leq C \int_{-\infty}^{\infty} c(u)\left\|\mathcal{G}_{+}^{i u}\right\|_{p} \mathrm{~d} u
\end{aligned}
$$

as required.

To prove (ii), first we observe that it is not very hard to check that the conclusion of [11, Theorem 4] holds under the slightly weaker assumption that the multiplier $m$ (we keep the notation of [11]) satisfies Hörmander like conditions (1.1) of nonintegral order $\alpha>\beta / 2+1$, instead of a corresponding Hörmander condition of integral order $\alpha>\beta / 2+1$ (see the beginning of [11, Section 2] for the definition).

Then the proof of (ii) is, mutatis mutandis, the same as the proof of [7, Theorem 2.2]. Note however that (ii) is not a corollary of [7, Theorem 2.2], for the family of operators $\int_{0}^{\infty} e^{-t \zeta} \mathrm{d} \mathcal{E}_{\zeta}$ is neither a strongly continuous semigroup of operators on $L^{p}(X)$, nor a family of positive operators on the Banach lattice $L^{2}(X)$. 


\section{3. - Functional calculus of Laplace transform type for $\mathcal{L}_{b}$}

Suppose that $p$ is in $(1, \infty)$ and that $b$ is in $[0, \infty)$. In this section we shall prove that the operator $\mathcal{L}_{b}$ has functional calculus of Laplace transform type in $L^{p}(\gamma)$ (see Theorem 3.2).

Let $\Pi_{b}$ denote the projector $\sum_{j>b} \mathcal{P}_{j}$. Since $\Pi_{b}=\mathcal{I}-\sum_{j \leq b} \mathcal{P}_{j}$ and each $\mathcal{P}_{j}$ extends to a bounded operator on $L^{p}(\gamma)$, we conclude that $\Pi_{b}$ also extends to a bounded operator on $L^{p}(\gamma)$.

First, we prove a technical lemma, of independent interest, concerning estimates of the norm of the operator $t \mathcal{L}_{b} e^{-t \mathcal{L}_{b}} \Pi_{b}$ in $L^{p}(\gamma)$.

Lemma 3.1. Suppose that $p$ is in $(1, \infty) \backslash\{2\}$, that $b$ is in $[0, \infty)$, and that $\theta$ is in $(0, \pi / 2)$. Set $\delta_{b}=[b]+1-b$ and $t_{p}=-\log \sqrt{\min \left(p-1, p^{\prime}-1\right)}$. Then there exists a constant $C$ such that for every $z$ in $\mathbf{E}_{p} \cap \mathbf{S}_{\theta}$ with $\operatorname{Re} z \geq 2 t_{p}$

$$
\left\|z \mathcal{L}_{b} e^{-z \mathcal{L}_{b}} \Pi_{b}\right\|_{p} \leq C e^{-\delta_{b} \operatorname{Re} z / 2} .
$$

Proof. Throughout the proof of this lemma we shall denote by $t$ the real part of $z$. A duality argument shows that it suffices to prove the lemma in the case where $p$ is in $(1,2)$.

Recall that by Nelson's result [12] $t_{p}$ is the minimum of all $t$ such that $e^{-t \mathcal{L}}$ maps $L^{p}(\gamma)$ into $L^{2}(\gamma)$. By arguing much as in [7, Proposition 3.2], we get

$$
\begin{aligned}
\left\|z \mathcal{L}_{b} e^{-z \mathcal{L}_{b}} \Pi_{b} f\right\|_{p} & \leq\left\|z \mathcal{L}_{b} e^{-z \mathcal{L}_{b}} \Pi_{b} f\right\|_{2} \\
& =\left\|\sum_{j>b} z(j-b) e^{-z(j-b)} \mathcal{P}_{j} f\right\|_{2} \\
& =\left(\sum_{j>b}|z|^{2}(j-b)^{2} e^{-2 t(j-b)}\left\|\mathcal{P}_{j} f\right\|_{2}^{2}\right)^{1 / 2} .
\end{aligned}
$$

Since $z$ is in $\mathbf{S}_{\theta},|z|^{2} \leq(1+\tan \theta)^{2} t^{2}$. Furthermore, there exists a constant $C$ such that $x^{2} e^{-2 x} \leq C e^{-x}$ on $\mathbb{R}^{+}$. Thus,

$$
\begin{aligned}
& \left\|z \mathcal{L}_{b} e^{-z \mathcal{L}_{b}} \Pi_{b} f\right\|_{p} \leq C\left(\sum_{j>b} e^{-t(j-b)}\left\|\mathcal{P}_{j} f\right\|_{2}^{2}\right)^{1 / 2} \\
& =C\left(\sum_{j>b} e^{-\left(t-2 t_{p}+2 t_{p}\right)(j-b)}\left\|\mathcal{P}_{j} f\right\|_{2}^{2}\right)^{1 / 2} \\
& \leq C e^{-\delta_{b}\left(t-2 t_{p}\right) / 2}\left(\sum_{j>b} e^{-2 t_{p}(j-b)}\left\|\mathcal{P}_{j} f\right\|_{2}^{2}\right)^{1 / 2} \\
& \leq C e^{-\delta_{b} t / 2}\left(\sum_{j>b} e^{-2 t_{p} j}\left\|\mathcal{P}_{j} f\right\|_{2}^{2}\right)^{1 / 2} \\
& \leq C e^{-\delta_{b} t / 2}\left\|e^{-t_{p} \mathcal{L}} f\right\|_{2} \\
& \text { (by Nelson's result) } \quad \leq C e^{-\delta_{b} t / 2}\|f\|_{p} \quad \forall f \in L^{2}(\gamma) \cap L^{p}(\gamma) \quad \forall t \in\left[2 t_{p}, \infty\right) \text {. }
\end{aligned}
$$


A density argument then shows that if $z$ is in $\mathbf{S}_{\theta}$, then

$$
\left\|z \mathcal{L}_{b} e^{-z \mathcal{L}_{b}} \Pi_{b}\right\|_{p} \leq C e^{-\delta_{b} t / 2} \quad \forall t \in\left[2 t_{p}, \infty\right),
$$

as required.

THeOREM 3.2. Suppose that $p$ is in $(1, \infty) \backslash\{2\}$, and that $b$ is in $[0, \infty)$. Then the operator $\mathcal{L}_{b}$ admits a functional calculus of Laplace transform type in $L^{p}(\gamma)$.

PROOF. A duality argument shows that it suffices to prove the required result for $p$ in $(1,2)$.

Suppose that $M$ vanishes in $(-\infty, 0]$ and is of Laplace transform type as in (2.1). By spectral theory

$$
M\left(\mathcal{L}_{b}\right)=\int_{0}^{\infty} m(s) \mathcal{L}_{b} e^{-s \mathcal{L}_{b}} \Pi_{b} \mathrm{~d} s,
$$

where the integral converges in the weak operator topology of $L^{2}(\gamma)$. Observe that

$$
\begin{aligned}
\left\|\int_{2 t_{p}}^{\infty} m(s) \mathcal{L}_{b} e^{-s \mathcal{L}_{b}} \Pi_{b} \mathrm{~d} s\right\|_{p} & \leq\|m\|_{\infty} \int_{2 t_{p}}^{\infty}\left\|s \mathcal{L}_{b} e^{-s \mathcal{L}_{b}} \Pi_{b}\right\|_{p} \frac{\mathrm{d} s}{s} \\
(\text { by Lemma 3.1) } & \leq\|m\|_{\infty} \int_{2 t_{p}}^{\infty} e^{-\delta_{b} s} \frac{\mathrm{d} s}{s} \\
& \leq C\|m\|_{\infty} .
\end{aligned}
$$

Thus, to conclude the proof of the theorem it suffices to show that there exists a constant $C$ such that

$$
\left\|\int_{0}^{2 t_{p}} m(s) \mathcal{L}_{b} e^{-s \mathcal{L}_{b}} \Pi_{b} \mathrm{~d} s\right\|_{p} \leq C\|m\|_{\infty} .
$$

Observe that

$$
\int_{0}^{2 t_{p}} m(s) \mathcal{L}_{b} e^{-s \mathcal{L}_{b}} \Pi_{b} \mathrm{~d} s=\int_{0}^{2 t_{p}} e^{b s} m(s) \mathcal{L} e^{-s \mathcal{L}} \Pi_{b} \mathrm{~d} s-b \int_{0}^{2 t_{p}} e^{b s} m(s) e^{-s \mathcal{L}} \Pi_{b} \mathrm{~d} s .
$$

Clearly

$$
\left\|\int_{0}^{2 t_{p}} e^{b s} m(s) e^{-s \mathcal{L}} \Pi_{b} \mathrm{~d} s\right\|_{p} \leq e^{2 b t_{p}}\|m\|_{\infty}\left\|\Pi_{b}\right\|_{p} \int_{0}^{2 t_{p}}\left\|e^{-s \mathcal{L}_{1}}\right\|_{p} \mathrm{~d} s
$$

$\left(e^{-s \mathcal{L}}\right.$ is contractive on $\left.L^{p}(\gamma)\right) \leq 2 t_{p} e^{2 b t_{p}}\|m\|_{\infty}\left\|\Pi_{b}\right\|_{p}$.

Furthermore

$$
\begin{aligned}
\left\|\int_{0}^{2 t_{p}} e^{b s} m(s) \mathcal{L} e^{-s \mathcal{L}} \Pi_{b} \mathrm{~d} s\right\|_{p} & =\left\|\int_{0}^{2 t_{p}} e^{b s} m(s) \mathcal{L} e^{-s \mathcal{L}} \mathrm{d} s \Pi_{b}\right\|_{p} \\
& \leq C\left\|\int_{0}^{2 t_{p}} e^{b s} m(s) \mathcal{L} e^{-s \mathcal{L}} \mathrm{d} s\right\|_{p}
\end{aligned}
$$


Now observe that the operator $\int_{0}^{2 t p} e^{b s} m(s) \mathcal{L} e^{-s \mathcal{L}} \mathrm{d} s$ is of Laplace transform type, and corresponds to the $L^{\infty}\left(\mathbb{R}^{+}\right)$function $m_{1}$ defined by

$$
m_{1}(s)=e^{b s} m(s) \mathbf{1}_{\left[0,2 t_{p}\right]}(s) \quad \forall s \in \mathbb{R}^{+} .
$$

Therefore [13, Cor. 3] (or [1]) implies that

$$
\begin{aligned}
\left\|\int_{0}^{2 t_{p}} e^{b s} m(s) \mathcal{L} e^{-s \mathcal{L}} \mathrm{d} s\right\|_{p} & \leq C\left\|m_{1}\right\|_{\infty} \\
& \leq C\|m\|_{\infty},
\end{aligned}
$$

concluding the proof of the theorem.

\section{4. - Estimates for imaginary powers of $\mathcal{L}_{b}$}

In this section we improve the estimates of $\left\|\left(\mathcal{L}_{b}\right)_{+}^{i u}\right\|_{p}$ obtained in [7, formula (2)]. The strategy we adopt is similar to that of [7, Proposition 3.1 and Proposition 3.2], where uniform bounds of $\left\|(\mathcal{L}+\varepsilon \mathcal{I})^{i u}\right\|_{p}$, for $\varepsilon \in(0,1]$, were obtained. However, there are significant differences.

REMARK 4.1. Recall that for every $p$ in $(1, \infty) \backslash\{2\}$ the Ornstein-Uhlenbeck semigroup $\left\{e^{-t \mathcal{L}}\right\}_{t \geq 0}$ extends to a contraction semigroup on $L^{p}(\gamma)$ in the Epperson region $\mathbf{E}_{p}[5]$, where

$$
\mathbf{E}_{p}=\left\{x+i y \in \mathbb{C}:|\sin y| \leq \tan \phi_{p} \sinh x\right\}
$$

and $\phi_{p}=\arccos |2 / p-1|$. In [7, Section 3] the authors made use of the transformation $\tau:(\mathbb{C} \backslash \mathbb{R}) \cup(-1,1) \rightarrow \mathbb{C}$, defined by

$$
\tau(w)=\log \frac{1+w}{1-w},
$$

where $\log w$ is real when $w>0$, to straighten the part of the boundary of $\mathbf{E}_{p}$ close to the origin. Indeed, $\tau$ maps $\mathbf{S}_{\phi_{p}} \backslash[1, \infty)$ onto the interior of $\mathbf{E}_{p} \cap\{z \in \mathbb{C}:|\operatorname{Im} z|<\pi\}$ and the ray $\mathbb{R}^{+} e^{i \phi_{p}}$ onto $\partial \mathbf{E}_{p} \cap\{z \in \mathbb{C}: 0<\operatorname{Im} z<\pi\}$. We denote by $z_{p}$ the point $\tau\left(e^{i \phi_{p}} / 2\right)$, which is on the boundary of $\mathbf{E}_{p}$.

We consider a path in $\mathbf{E}_{p}$ which starts at the origin and runs along the the boundary of $\mathbf{E}_{p}$ up to the point $z_{p}$. To be more specific, let $\alpha_{p}:[0,1 / 2] \rightarrow \mathbb{C}$ be the $\operatorname{path}^{(1)} \alpha_{p}(t)=\tau\left(e^{i \phi_{p}} t\right)$

${ }^{(1)}$ We take the opportunity to correct a misprint in the definition of $\alpha_{p}$ in [7, beginning of $\left.\mathrm{p} .426\right]$. The path $\alpha_{p}$ is the map $t \mapsto \tau\left(e^{i \phi_{p}} t\right)$, not $t \mapsto e^{i \phi_{p}} t$, as erroneously written there. 
For every $u$ in $\mathbb{R}$ let $J^{p, i u}: \mathbb{R} \rightarrow \mathbb{C}$ be the function which vanishes on $(-\infty, 0]$, and is defined on $\mathbb{R}^{+}$by

$$
J^{p, i u}(\zeta)=\frac{\zeta}{\Gamma(1+i u)} \int_{\alpha_{p}} z^{i u} e^{-\zeta z} \mathrm{~d} z+\frac{z_{p}^{i u}}{\Gamma(1+i u)} e^{-z p \zeta} .
$$

Remark 4.2. The restriction of $J^{p, i u}$ to $\mathbb{R}^{+}$agrees with [7, formula (8)]. For every $\varepsilon$ in $\mathbb{R}$, let $r_{\varepsilon}^{p, i u}$ denote the smooth function defined on the complement of the diagonal in $\mathbb{R}^{d^{\varepsilon}} \times \mathbb{R}^{d}$ by

$$
r_{\varepsilon}^{p, i u}(x, y)=\frac{e^{\left(|x|^{2}+|y|^{2}\right) / 2}}{2^{d} \Gamma(i u)} \int_{\tau^{-1} \circ \alpha_{p}} w^{-d / 2-1} g_{\varepsilon}(w) e^{-\frac{1}{4}\left[w|x+y|^{2}+w^{-1}|x-y|^{2}\right]+i u \log \tau(w)} \mathrm{d} w
$$

where

$$
g_{\varepsilon}(w)=w \frac{(1+w)^{d}}{\tau(w)} e^{-\varepsilon \tau(w)} \tau^{\prime}(w) .
$$

In [7, Proposition 5.2] the authors proved that if $\varepsilon$ is in $(0,1]$, then $J^{p, i u}(\mathcal{L}+\varepsilon \mathcal{I})$ is a singular integral operator and

$$
J^{p, i u}(\mathcal{L}+\varepsilon \mathcal{I}) f(x)=\int r_{\varepsilon}^{p, i u}(x, y) f(y) \mathrm{d} \gamma(y) \quad \forall f \in C_{c}^{\infty}\left(\mathbb{R}^{d}\right) \quad \forall x \notin \operatorname{supp}(f) .
$$

Thus, the kernel of $J^{p, i u}(\mathcal{L}+\varepsilon \mathcal{I})$ agrees with $r_{\varepsilon}^{p, i u}$ off the diagonal of $\mathbb{R}^{d} \times \mathbb{R}^{d}$. It is not hard to show that the same holds for all $\varepsilon$ in $\mathbb{R}$, i.e., that $J^{p, i u}(\mathcal{L}+\varepsilon \mathcal{I})$ is a singular integral operator whose kernel coincides with $r_{\varepsilon}^{p, i u}$ off the diagonal of $\mathbb{R}^{d} \times \mathbb{R}^{d}$.

THEOREM 4.3. Suppose that $p$ is in $(1, \infty) \backslash\{2\}$ and that $b$ is in $[0, \infty)$. Then there exists a constant $C$ such that

$$
\left\|\left(\mathcal{L}_{b}\right)_{+}^{i u}\right\|_{p} \leq C e^{\phi_{p}^{*}|u|} \quad \forall u \in \mathbb{R} .
$$

Proof. Since $\mathcal{L}_{b}$ preserves the class of real functions, it suffices to prove the required estimate in the case where $u$ is positive. A proof of this fact may be found in [7, Remark 2.4].

Let $\omega_{p}:[1, \infty] \rightarrow \mathbb{C}$ denote the path $\omega_{p}(t)=z_{p} t$. Note that the path $\alpha_{p}+\omega_{p}\left(\alpha_{p}\right.$ is defined in Remark 4.1) starts at the origin, runs along the boundary of $\mathbf{E}_{p}$ up to the point $z_{p}$ and then goes to $\infty$ along the ray $\mathbb{R} z_{p}$. Observe that

$$
\begin{aligned}
& \zeta^{-i u}=\frac{\zeta}{\Gamma(1+i u)} \int_{0}^{\infty} s^{i u} e^{-\zeta s} \mathrm{~d} s \\
& \text { (by Cauchy's theorem) } \quad=\frac{\zeta}{\Gamma(1+i u)} \int_{\alpha_{p}+\omega_{p}} z^{i u} e^{-\zeta z} \mathrm{~d} z \quad \forall \zeta \in \mathbb{R}^{+} .
\end{aligned}
$$


For every real number $u$, let $A_{p, u}, B_{p, u}: \mathbb{R} \rightarrow \mathbb{C}$ be the functions which vanish on $(-\infty, 0]$ and are defined on $\mathbb{R}^{+}$by

$$
A_{p, u}(\zeta)=\frac{\zeta}{\Gamma(1+i u)} \int_{\alpha_{p}} z^{i u} e^{-\zeta z} \mathrm{~d} z \quad \text { and } \quad B_{p, u}(\zeta)=\frac{\zeta}{\Gamma(1+i u)} \int_{\omega_{p}} z^{i u} e^{-\zeta z} \mathrm{~d} z
$$

By the spectral theorem

$$
\left(\mathcal{L}_{b}\right)_{+}^{i u}=A_{p, u}\left(\mathcal{L}_{b}\right)+B_{p, u}\left(\mathcal{L}_{b}\right) .
$$

We shall estimate separately the norms of $A_{p, u}\left(\mathcal{L}_{b}\right)$ and of $B_{p, u}\left(\mathcal{L}_{b}\right)$ as operators on $L^{p}(\gamma)$.

First we estimate $\left\|B_{p, u}\left(\mathcal{L}_{b}\right)\right\|_{p}$. Observe that $\left|\omega_{p}(t)^{i u}\right|=e^{-u \arg z p}$ for all $t \geq 1$, so that

$$
\left\|B_{p, u}\left(\mathcal{L}_{b}\right)\right\|_{p} \leq \frac{e^{-u \arg z p}}{|\Gamma(1+i u)|} \int_{\omega_{p}}\left\|z \mathcal{L}_{b} e^{-z \mathcal{L}_{b}} \Pi_{b}\right\|_{p}\left|\frac{\mathrm{d} z}{z}\right|
$$

(by Lemma 3.1 and Stirling's formula) $\leq C \frac{e^{(\pi / 2-\arg z p) u}}{(1+u)^{1 / 2}}$

$$
\text { (since } \left.\arg z_{p}>\phi_{p}\right) \quad \leq C e^{\left(\phi_{p}^{*}-\varepsilon\right) u} \quad \forall u \in \mathbb{R}^{+}
$$

for some positive $\varepsilon$.

Next we estimate $\left\|A_{p, u}\left(\mathcal{L}_{b}\right)\right\|_{p}$. Note that

$$
A_{p, u}\left(\mathcal{L}_{b}\right)=J^{p, i u}\left(\mathcal{L}_{b}\right)-\frac{z_{p}^{i u}}{\Gamma(1+i u)} e^{-z_{p} \mathcal{L}_{b}} \Pi_{b},
$$

where $J^{p, i u}$ is defined in (4.1).

Observe that $\Pi_{b}$ is bounded on $L^{p}(\gamma)$, that $e^{-z \mathcal{L}}$ is contractive for all $z$ in $\mathbf{E}_{p}$ and that $\arg z_{p}>\phi_{p}$. These facts and Stirling's formula imply that

$$
\left\|\frac{z_{p}^{i u}}{\Gamma(1+i u)} e^{-z_{p} \mathcal{L}_{b}} \Pi_{b}\right\|_{p} \leq C e^{\phi_{p}^{*} u} \quad \forall u \in \mathbb{R}^{+} .
$$

Therefore, to conclude the proof of the theorem, it suffices to show that

$$
\left\|J^{p, i u}\left(\mathcal{L}_{b}\right)\right\|_{p} \leq C e^{\phi_{p}^{*} u} \quad \forall u \in \mathbb{R}^{+} .
$$

By Remark 4.2 (with $-b$ in place of $\varepsilon$ ) the kernel of $J^{p, i u}\left(\mathcal{L}_{b}\right)$ agrees with $r_{-b}^{p, i u}$ off the diagonal (see (4.2)). Following the approach used in previous papers on the subject [7], [6], to estimate $\left\|J^{p, i u}\left(\mathcal{L}_{b}\right)\right\|_{p}$ we decompose $J^{p, i u}\left(\mathcal{L}_{b}\right)$ as the sum of a "local part" and the remaining "global part". Specifically, we consider the set

$$
L=\left\{(x, y) \in \mathbb{R}^{d} \times \mathbb{R}^{d}:|x-y| \leq \min \left(1,|x+y|^{-1}\right)\right\},
$$


and denote by $G$ its complement in $\mathbb{R}^{d} \times \mathbb{R}^{d}$. We shall call $L$ and $G$ the local and the global region, respectively. Let $\phi$ be a smooth function on $\mathbb{R}^{d} \times \mathbb{R}^{d}$ which is equal to 1 in $L$, vanishes off

$$
\left\{(x, y) \in \mathbb{R}^{d} \times \mathbb{R}^{d}:|x-y| \leq 2 \min \left(1,|x+y|^{-1}\right)\right\},
$$

and satisfies the estimate

$$
\left|\nabla_{x} \phi(x, y)\right|+\left|\nabla_{y} \phi(x, y)\right| \leq C|x-y|^{-1} .
$$

The global part $J_{\text {glob }}^{p, i u}\left(\mathcal{L}_{b}\right)$ of the operator $J^{p, i u}\left(\mathcal{L}_{b}\right)$ is the integral operator defined by

$$
J_{\text {glob }}^{p, i u}\left(\mathcal{L}_{b}\right) f(x)=\int_{\mathbb{R}^{d}} r_{-b}^{p, i u}(x, y)(1-\phi(x, y)) f(y) \mathrm{d} \gamma(y) \quad \forall f \in C_{c}^{\infty}\left(\mathbb{R}^{d}\right) .
$$

The local part $J_{\text {loc }}^{p, i u}\left(\mathcal{L}_{b}\right)$ of $J^{p, i u}\left(\mathcal{L}_{b}\right)$ is then defined by

$$
J_{\text {loc }}^{p, i u}\left(\mathcal{L}_{b}\right) f=J^{p, i u}\left(\mathcal{L}_{b}\right) f-J_{\text {glob }}^{p, i u}\left(\mathcal{L}_{b}\right) f \quad \forall f \in C_{c}^{\infty}\left(\mathbb{R}^{d}\right) .
$$

It is easy to show that $J_{\text {loc }}^{p, i u}\left(\mathcal{L}_{b}\right)$ is a singular integral operator whose kernel agrees with $r_{-b}^{p, i u} \phi$ off the diagonal.

Now we estimate $\left\|J_{\text {loc }}^{p, i u}\left(\mathcal{L}_{b}\right)\right\|_{p}$. By [6, Theorem 3.7], $\left\|J_{\text {loc }}^{p, i u}\left(\mathcal{L}_{b}\right)\right\|_{p}$ is bounded by a constant times the sum of $\left\|J^{p, i u}\left(\mathcal{L}_{b}\right)\right\|_{2}$ and the constants appearing in the standard Calderón-Zygmund estimates of the kernel $r_{-b}^{p, i u} \phi$ in the local region $L$. To obtain these Calderón-Zygmund estimates we argue as in [7, Proposition 4.1 and Lemma 5.1], but with a twist: in the integrals defining the operator $J^{p, i u}\left(\mathcal{L}_{b}\right)$ and its kernel $r_{-b}^{p, i u}$ we use the path $\tilde{\alpha}_{p}(t)=t z_{p}$, for $0<t \leq 1$, instead of the path $\alpha_{p}(t)=\tau\left(t e^{i \phi_{p}}\right)$, for $0<t \leq 1 / 2$. The integrals do not change because the integrands are holomorphic. Since $\left|\tilde{\alpha}_{p}(t)^{i u}\right|=e^{-\left(\phi_{p}+\epsilon_{p}\right) u}$, because $\arg z_{p}=\phi_{p}+\epsilon_{p}$, for some $\epsilon_{p}>0$, one obtains an additional factor $e^{-\epsilon_{p} u}$ both in the estimate of the norm on $L^{2}(\gamma)$ of the operator and in the standard Calderón-Zygmund estimates of its kernel. This extra exponential decay when $u$ tends to infinity is more than enough to cancel the polynomial factor in the estimate

$$
\left\|J_{\text {loc }}^{p, i u}(\mathcal{L}+\varepsilon \mathcal{I})\right\|_{p} \leq C(1+u)^{5 / 2} e^{\phi_{p}^{*} u} \quad \forall u \in \mathbb{R}^{+} \quad \forall \varepsilon \in(0,1],
$$

obtained in [7, Proposition 3.1]. Therefore

$$
\left\|J_{\text {loc }}^{p, i u}\left(\mathcal{L}_{b}\right)\right\|_{p} \leq C e^{\phi_{p}^{*} u} \quad \forall u \in \mathbb{R}^{+} .
$$


It remains to estimate the norm of $J_{\text {glob }}^{p, i u}\left(\mathcal{L}_{b}\right)$ on $L^{p}(\gamma)$. Since the cut-off $1-\phi$ is supported in the global region $G$, it follows from the definition of $J_{\text {glob }}^{p, i u}\left(\mathcal{L}_{b}\right)$ given in (4.6) that

$$
\left|J_{\text {glob }}^{p, i u}\left(\mathcal{L}_{b}\right) f(x)\right| \leq \int_{G_{x}}\left|r_{-b}^{p, i u}(x, y)\right||f(y)| \mathrm{d} \gamma(y),
$$

where $G_{x}$ is the $x$-section of the set $G$. We need to improve the estimate of the growth of $\left|r_{\varepsilon}^{p, i u}(x, y)\right|$ as $u$ tends to infinity obtained in [7, Proposition 4.3].

The expression (4.2) for the kernel $r_{-b}^{p, i u}(x, y)$ contains an oscillatory integral. To write it in a more compact form, we introduce the following notation. For each function $g$ analytic in the unit disk, consider the oscillatory integral $I(u, v, \sigma ; g)$, depending on the parameters $u, v, \sigma$ and defined by

$$
I(u, v, \sigma ; g)=\int_{0}^{1 /(2 \sigma)} t^{-d / 2-1} g(\sigma t) e^{u F(t, v, \sigma)} \mathrm{d} t
$$

where

$$
F(t, v, \sigma)=-v\left(t^{-1}+t-2\right)+i\left(\tan \phi_{p}\right) v\left(t^{-1}-t\right)+i \log \left(\tau\left(\sigma t e^{i \phi_{p}}\right)\right)+\phi_{p} .
$$

If we set

$$
\begin{aligned}
\tilde{g}_{-b}(t) & =g_{-b}\left(t e^{i \phi_{p}}\right), \\
a(x, y) & =\frac{\cos \phi_{p}}{4}|x+y||x-y|, \\
\sigma(x, y) & =|x-y| /|x+y|, \\
Q_{p}(x, y) & =\frac{1}{2}\left(|x|^{2}+|y|^{2}-\cos \phi_{p}|x+y||x-y|\right),
\end{aligned}
$$

it is straightforward to see that

$$
r_{-b}^{p, i u}(x, y)=\frac{e^{-i \phi_{p} d / 2}}{2^{d} \Gamma(i u)} e^{-u \phi_{p}} \sigma(x, y)^{-d / 2} I\left(u, \frac{a(x, y)}{u}, \sigma(x, y) ; \tilde{g}_{-b}\right) e^{Q_{p}(x, y)} .
$$

Hence,

$$
\left|r_{-b}^{p, i u}(x, y)\right| \leq C k_{-b}^{p, i u}(x, y) e^{Q_{p}(x, y)},
$$

where

$$
k_{-b}^{p, i u}(x, y)=(1+|u|)^{1 / 2} e^{\phi_{p}^{*} u} \sigma(x, y)^{-d / 2}\left|I\left(u, \frac{a(x, y)}{u}, \sigma(x, y) ; \tilde{g}_{-b}\right)\right| .
$$

Note that we have used Stirling's formula and that $k_{-b}^{p, i u}(x, y)$ is symmetric in $x$ and $y$. Thus, by (4.8),

$$
\left|J_{\text {glob }}^{p, i u}\left(\mathcal{L}_{b}\right) f(x)\right| \leq C \int_{G_{x}} k_{-b}^{p, i u}(x, y) e^{Q_{p}(x, y)}|f(y)| \mathrm{d} \gamma(y) \quad \forall f \in C_{c}^{\infty}\left(\mathbb{R}^{d}\right) .
$$


To estimate the norm on $L^{p}(\gamma)$ of the operator

$$
\mathcal{T} f(x)=\int_{G_{x}} k_{-b}^{p, i u}(x, y) e^{Q_{p}(x, y)}|f(y)| \mathrm{d} \gamma(y) \quad \forall f \in C_{c}^{\infty}\left(\mathbb{R}^{d}\right),
$$

we use the isometry $\mathcal{U}_{p}: L^{p}(\lambda) \rightarrow L^{p}(\gamma)$ defined by $\mathcal{U}_{p} f(x)=e^{|x|^{2} / p} f(x)$, and estimate the norm on $L^{p}(\lambda)$ of the conjugate operator $\mathcal{U}_{p}^{-1} \mathcal{T} \mathcal{U}_{p}$.

It is easy to check that the kernel of this conjugate operator with respect to Lebesgue measure is

$$
k_{-b}^{p, i u}(x, y) e^{Q_{p}(x, y)-|x|^{2} / p-|y|^{2} / p^{\prime}} \leq k_{-b}^{p, i u}(x, y) e^{q_{p}(x, y)},
$$

where

$$
q_{p}(x, y)=\frac{\cos \phi_{p}}{2}\left(\left.|| x\right|^{2}-|y|^{2}|-| x+y|| x-y \mid\right) .
$$

We claim that the following is true:

$$
\sup _{x \in \mathbb{R}^{d}} \int_{G_{x}} k_{-b}^{p, i u}(x, y) e^{q_{p}(x, y)} \mathrm{d} y \leq C e^{\phi_{p}^{*} u} \quad \forall u \geq 1 .
$$

Assuming this claim for the moment, we complete the proof. The claim implies that the norm of the integral operator with kernel $k_{-b}^{p, i u}(x, y) e^{q_{p}(x, y)}$ on $L^{\infty}(\lambda)$ is bounded by $C e^{\phi_{p}^{*} u}$. Since the kernel is symmetric, the same bound holds for the norm on $L^{1}(\lambda)$ and, by interpolation, on $L^{p}(\lambda)$. This proves that

$$
\left\|J_{\text {glob }}^{p, i u}\left(\mathcal{L}_{b}\right)\right\|_{p} \leq C e^{\phi_{p}^{*} u} \quad \forall u \geq 1 .
$$

By [7, Proposition 3.1] the same estimate holds also for $0<u<1$.

Now, (4.7) and (4.12) imply (4.5). Thus, to complete the proof of Theorem 4.3, we only need to prove claim (4.11). This we shall do in Proposition 5.5 below.

\section{5. - Proof of claim (4.11)}

To prove claim (4.11), we need to estimate the oscillatory integral $I(u, \nu, \sigma ; g)$, defined in (4.9), for $u \geq 1$. For the reader's convenience we recall that

$$
I(u, v, \sigma ; g)=\int_{0}^{1 /(2 \sigma)} t^{-d / 2-1} g(\sigma t) e^{u F(t, v, \sigma)} \mathrm{d} t,
$$

where the function $g$ is analytic in the unit disk, $F$ is given by

$$
F(t, v, \sigma)=-v\left(t^{-1}+t-2\right)+i\left(\tan \phi_{p}\right) v\left(t^{-1}-t\right)+i \log \left(\tau\left(\sigma t e^{i \phi_{p}}\right)\right)+\phi_{p},
$$

and $\tau(w)=\log (1+w)-\log (1-w)$, as before (see Remark 4.1). 
The function $F$ is smooth in the set $\{(t, v, \sigma) \in \mathbb{R} \times \mathbb{R} \times \mathbb{R}: t>0, \sigma>$ $0, t \sigma<1\}$. For our purposes, it is convenient to replace $F$ with the function

$$
\Phi(t, v, \sigma)=F(t, v, \sigma)-i \log \sigma .
$$

which extends to a smooth function in a neighbourhood of each point $(1, v, 0)$, as we shall prove below (see Remark 5.2). Note that

$$
I(u, v, \sigma ; g)=e^{i u \log \sigma} \int_{0}^{1 /(2 \sigma)} t^{-d / 2-1} g(\sigma t) e^{u \Phi(t, v, \sigma)} \mathrm{d} t .
$$

For the sake of brevity, we write $v_{p}=\left(2 \tan \phi_{p}\right)^{-1}$ and

$$
\varphi(t)=\log \left(\tau\left(t e^{i \phi_{p}}\right)\right)-i \phi_{p},
$$

so that

$$
\Phi(t, v, \sigma)=-v\left(t^{-1}+t-2\right)+i \frac{v}{2 v_{p}}\left(t^{-1}-t\right)+i(\varphi(\sigma t)-\log \sigma) .
$$

Lemma 5.1. The following expansions hold

$$
\begin{aligned}
\tau(w) & =2 w+\frac{2}{3} w^{3}+O\left(w^{5}\right) \\
\varphi(t) & =\log 2+\log t+\frac{1}{3} t^{2} e^{2 i \phi_{p}}+O\left(t^{4}\right) \\
\varphi^{\prime}(t) & =\frac{1}{t}+\frac{2}{3} t e^{2 i \phi_{p}}+O\left(t^{3}\right) \\
\varphi^{\prime \prime}(t) & =-\frac{1}{t^{2}}+\frac{2}{3} e^{2 i \phi_{p}}+O\left(t^{2}\right),
\end{aligned}
$$

as $w$ and $t$ tend to 0 . Moreover the function $(t, \sigma) \mapsto \varphi(\sigma t)-\log \sigma$ extends to a smooth function in a neighbourhood of $(1,0)$.

PROOF. The first two expansions follow from the McLaurin expansion of $w \mapsto \log (1+w)$. The last two expansions are obtained by differentiating (5.4). The last statement is a straightforward consequence of (5.4).

REMARK 5.2. The function $\Phi$ extends to a smooth function in a neighbourhood of each point $(1, v, 0)$, by (5.2) and Lemma 5.1. For future reference, we give here the expressions for the first and second derivatives of $\Phi$ with respect to $t$ :

$$
\begin{aligned}
\Phi_{t}^{\prime}(t, v, \sigma) & =v\left(t^{-2}-1\right)-i \frac{v}{2 v_{p}}\left(t^{-2}+1\right)+i \sigma \varphi^{\prime}(\sigma t), \\
\Phi_{t t}^{\prime \prime}(t, v, \sigma) & =-2 v t^{-3}+i \frac{v}{v_{p}} t^{-3}+i \sigma^{2} \varphi^{\prime \prime}(\sigma t) .
\end{aligned}
$$

Observe that $\operatorname{Re} \Phi\left(1, v_{p}, 0\right)=0, \Phi_{t}^{\prime}\left(1, v_{p}, 0\right)=0$ and $\Phi_{t t}^{\prime \prime}\left(1, v_{p}, 0\right)=-2 v_{p} \neq 0$. 
Lemma 5.3. There exists a constant $c_{p}>0$ such that

$$
\begin{aligned}
& \phi_{p}+c_{p} \min \left(1, t^{2}\right) \leq \arg \left(\tau\left(t e^{i \phi_{p}}\right)\right)<\pi / 2 \\
& \operatorname{Re} \Phi(t, v, \sigma) \leq-\frac{v}{t}(t-1)^{2}-c_{p} \min \left(1, \sigma^{2} t^{2}\right) \\
& \left|t \varphi^{\prime}(t)\right| \geq c_{p} \min \left(1, t^{-1}\right)
\end{aligned}
$$

for all $t>0, v>0$ and $\sigma \geq 0$. Moreover

$$
0 \leq \arg \left(\varphi^{\prime}(t)\right) \leq \pi / 2 \quad \forall t \in(0,1) .
$$

Proof. Let us write $\rho(t)=\left|\tau\left(t e^{i \phi_{p}}\right)\right|, \theta(t)=\arg \left(\tau\left(t e^{i \phi_{p}}\right)\right)$ and $y(t)=$ $\operatorname{Im}\left(\tau\left(t e^{i \phi_{p}}\right)\right)$, for the sake of brevity. We prove first that $y^{\prime}(t)>0, \theta^{\prime}(t)>0$ for all $t>0$ and $\rho^{\prime}(t)>0$ for all $t$ in $(0,1)$. Indeed, since $y(t)=\arg (1+$ $\left.t e^{i \phi_{p}}\right)-\arg \left(1-t e^{i \phi_{p}}\right)$, the inequality $y^{\prime}(t)>0$ for $t>0$ follows from a simple geometric consideration. Thus, as $t$ increases from 0 to $\infty$, the point $\tau\left(t e^{i \phi_{p}}\right)$ moves on the boundary curve of $\mathbf{E}_{p}$ from 0 to $i \pi$ (see Remark 4.1). This curve is the graph of the equation $x=f_{p}(y)$, where

$$
f_{p}(y)=\operatorname{arcsinh}\left(\frac{\sin y}{\tan \phi_{p}}\right), \quad 0 \leq y \leq \pi .
$$

It is easily verified that $f_{p}^{\prime \prime}<0$ in $[0, \pi]$, so that $x$ is a concave function of $y$. This implies that $\theta^{\prime}(t)>0$ for all $t>0$.

An elementary computation shows that

$$
\operatorname{Re} \tau\left(t e^{i \phi_{p}}\right)=\frac{1}{2} \log \left(1+\frac{1}{4 \cos \phi_{p}} \frac{t}{1+t^{2}-2 t \cos \phi_{p}}\right) .
$$

Since the $t$ derivative of the last fraction here is positive for $0<t<1$ and negative for $t>1$, the same is true for the $t$ derivative of $\operatorname{Re} \tau\left(t e^{i \phi_{p}}\right)$. It follows that $\rho^{\prime}(t)>0$ for $0<t<1$.

Now, to prove (5.8), we observe that by (5.3)

$$
\begin{aligned}
\arg \left(\tau\left(t e^{i \phi_{p}}\right)\right) & =\arctan \left(\frac{\operatorname{Im} \tau\left(t e^{i \phi_{p}}\right)}{\operatorname{Re} \tau\left(t e^{i \phi_{p}}\right)}\right) \\
& =\arctan \left(\tan \phi_{p}+\frac{\sin 2 \phi_{p}}{\cos ^{2} \phi_{p}} \frac{t^{2}}{3}+O\left(t^{4}\right)\right) \\
& =\phi_{p}+\left(\sin 2 \phi_{p}\right) \frac{t^{2}}{3}+O\left(t^{4}\right) \\
& \geq \phi_{p}+c t^{2},
\end{aligned}
$$


for some $c>0$, if $t$ is sufficiently small, say $t<t_{0}$. Since $\arg \left(\tau\left(t e^{i \phi_{p}}\right)\right)$ is strictly increasing in $(0, \infty)$, there exists $c^{\prime}>0$ such that

$$
\pi / 2>\arg \left(\tau\left(t e^{i \phi_{p}}\right)\right) \geq \phi_{p}+c^{\prime} \quad \forall t \geq t_{0} .
$$

By choosing $c_{p}=\min \left\{c, c^{\prime}\right\}$, we obtain (5.8).

To prove (5.9) we only need to observe that

$$
\begin{aligned}
\operatorname{Re} \Phi(t, v, \sigma) & =-\frac{v}{t}(t-1)^{2}-\operatorname{Im} \varphi(\sigma t) \\
& =-\frac{v}{t}(t-1)^{2}-\arg \left(\tau\left(\sigma t e^{i \phi_{p}}\right)\right)+\phi_{p}
\end{aligned}
$$

and apply (5.8). For (5.10) we simply observe that

$$
\varphi^{\prime}(t)=\frac{\tau^{\prime}\left(t e^{i \phi_{p}}\right)}{\tau\left(t e^{i \phi_{p}}\right)} e^{i \phi_{p}}=\frac{2}{\left(1-t^{2} e^{2 i \phi_{p}}\right) \tau\left(t e^{i \phi_{p}}\right)} e^{i \phi_{p}}
$$

Hence (5.10) follows, because $t \mapsto\left|t \varphi^{\prime}(t)\right|$ is a nonvanishing continuous function on $(0, \infty)$, which tends to 1 as $t$ tends to 0 and is asymptotic to $2 /(\pi t)$ as $t$ tends to $\infty$.

To prove (5.11) we recall that $\tau\left(t e^{i \phi_{p}}\right)=\rho(t) e^{i \theta(t)}$, so that

$$
\varphi^{\prime}(t)=(\log \rho(t)+i \theta(t))^{\prime}=\frac{\rho^{\prime}(t)}{\rho(t)}+i \theta^{\prime}(t) .
$$

Since $\rho(t), \rho^{\prime}(t)$ and $\theta^{\prime}(t)$ are positive for $t$ in $(0,1)$, it is clear that $\arg \varphi^{\prime}(t)$ is in $(0, \pi / 2)$.

Proposition 5.4. Let $v_{p}=\left(2 \tan \phi_{p}\right)^{-1}$. Then there exist an interval $J=$ $\left(v_{p}-\delta, v_{p}+\delta\right) \subset \mathbb{R}^{+}$and constants $\eta \in(0,3 / 4), c>0$, such that for all analytic functions $g$ in the unit disk satisfying $\max _{0 \leq j \leq 2 k} \max _{|w| \leq 1 / 2}\left|g^{(j)}(w)\right| \leq 1$ and for every positive integer $k$, the following estimates hold for all $u \geq 1$ and $v>0$ :

(i) if $0<\sigma<\eta$ then

$|I(u, v, \sigma ; g)| \leq C_{k}\left[\frac{e^{-c u v}}{u(u v)^{(1 \vee(d / 2))}}+\mathbf{1}_{J}(v)\left(u^{-1 / 2} e^{-c u\left(\left(v-v_{p}\right)^{2}+\sigma^{2}\right)}+u^{-k}\right)+\frac{\mathbf{1}_{J}(v)}{u^{3 / 2} v}\right] ;$

(ii) if $\eta \leq \sigma \leq 3 / 4$ then

$$
|I(u, v, \sigma ; g)| \leq C\left((u v)^{-d / 2} e^{-c u\left(v^{2 / 3}+v\right)}+e^{-c u} v^{-1}\right) ;
$$

(iii) if $\sigma>3 / 4$ then

$$
|I(u, v, \sigma ; g)| \leq C(u \nu)^{-d / 2} e^{-c u(v \sigma)^{2 / 3}} e^{-c u v \sigma} .
$$


Proof. Since, by (5.1)

$$
|I(u, v, \sigma ; g)|=\left|\int_{0}^{1 /(2 \sigma)} t^{-d / 2-1} g(\sigma t) e^{u \Phi(t, v, \sigma)} \mathrm{d} t\right|,
$$

it suffices to estimate this latter integral. The function $\Phi$ extends analytically in $t$ to a function which is smooth in a neighbourhood of $\left(1, v_{p}, 0\right)$ in $\mathbb{C} \times \mathbb{R} \times \mathbb{R}$, and by Remark 5.2 the point $\left(1, v_{p}, 0\right)$ is a real solution of the critical point equation

$$
\Phi_{t}^{\prime}(t, v, \sigma)=0
$$

such that $\Phi_{t t}^{\prime \prime}\left(1, v_{p}, 0\right) \neq 0$. Hence, by the complex implicit function theorem, for every $(\nu, \sigma)$ in a neighbourhood of $\left(v_{p}, 0\right)$ in $\mathbb{R} \times \mathbb{R}$ there exists a unique complex number $t(\nu, \sigma)$ in a neighbourhood of 1 in $\mathbb{C}$, such that $\Phi_{t}^{\prime}(t(\nu, \sigma), \nu, \sigma)=0$. In particular $t\left(v_{p}, 0\right)=1$. and

Since $\operatorname{Re} \Phi \leq 0$ in a neighbourhood of $\left(1, v_{p}, 0\right)$ in $\mathbb{R} \times \mathbb{R} \times \mathbb{R}$ by (5.9),

$$
\operatorname{Re} \Phi\left(1, v_{p}, 0\right)=0, \quad \Phi_{t}^{\prime}\left(1, v_{p}, 0\right)=0, \quad \Phi_{t t}^{\prime \prime}\left(1, v_{p}, 0\right) \neq 0,
$$

we may use the method of stationary phase with complex-valued phase depending on the parameters $(v, \sigma)$. We apply Theorem 7.7.12 in [10], with $u$ and $-i \Phi$ in place of $\omega$ and $f$, respectively (see also Theorem 7.7.5 in [10]). Thus there exist a small neighbourhood $\mathcal{U}$ of $\left(1, v_{p}, 0\right)$ in $\mathbb{R} \times \mathbb{R} \times \mathbb{R}$ and differential operators $L_{j}=L_{j, \Phi, v, \sigma}$ such that for every function $\Psi \in C_{c}^{\infty}(\mathcal{U})$ the integral

$$
\int_{0}^{\infty} \Psi(t, v, \sigma) e^{u \Phi(t, v, \sigma)} \mathrm{d} t
$$

has, for every positive integer $k$, the asymptotic expansion

$$
\left(\frac{2 \pi}{\left(\Phi_{t t}^{\prime \prime}\right)_{0}(\nu, \sigma)}\right)^{1 / 2} e^{u \Phi_{0}(v, \sigma)} u^{-1 / 2} \sum_{j=0}^{k-1}\left(L_{j} \Psi\right)_{0}(\nu, \sigma) u^{-j}+R_{k}(u, v, \sigma)
$$

for all $u>0$. Here, for a function $G(t, v, \sigma)$, the notation $G_{0}(\nu, \sigma)$ stands for a function of $(v, \sigma)$ only, which is in the same residue class modulo the ideal generated by $\Phi_{t}^{\prime}(t, v, \sigma)$ in the algebra of germs of functions smooth at $\left(1, v_{p}, 0\right)$. Moreover (5.12) does not depend on the choice of the representatives. Each $L_{j}$ is a linear differential operator in $t$ of order at most $2 j$, whose coefficients are rational functions of $\partial_{t}^{\ell} \Phi(t, v, \sigma), 2 \leq \ell \leq 2 j+2$, all with denominator $\left(\Phi_{t t}^{\prime \prime}(t, v, \sigma)\right)^{3 j}$. The remainder term satisfies the estimate

$$
\left|R_{k}(u, v, \sigma)\right| \leq C_{k}(\Phi)\|\Psi\|_{C^{2 k}(\mathcal{U})} u^{-k} .
$$

We claim that, if the function $(t, v, \sigma) \mapsto G(t, v, \sigma)$ extends analytically in $t$ to a function which is smooth in a neighbourhood of $\left(1, v_{p}, 0\right)$ in $\mathbb{C} \times \mathbb{R} \times \mathbb{R}$, then we may choose

$$
G_{0}(\nu, \sigma)=G(t(\nu, \sigma), \nu, \sigma)
$$


Indeed, by Taylor's formula

$$
G(t, v, \sigma)=G(t(v, \sigma), v, \sigma)+(t-t(\nu, \sigma)) R(t, v, \sigma)
$$

with $R$ smooth. Hence $G(t, v, \sigma)$ and $G(t(\nu, \sigma), v, \sigma)$ are in the same residue class modulo the ideal generated by $t-t(v, \sigma)$. Thus, to prove the claim, we only need to show that $t-t(v, \sigma)$ is in the ideal generated by $\Phi_{t}^{\prime}(t, v, \sigma)$. But this is obvious, because the function $(t-t(v, \sigma)) / \Phi_{t}^{\prime}(t, v, \sigma)$ is smooth since $\Phi_{t t}^{\prime \prime}(t(v, \sigma), v, \sigma) \neq 0$ for $(v, \sigma)$ sufficiently close to $\left(v_{p}, 0\right)$.

Now we consider three intervals

$$
I=(1-\epsilon, 1+\epsilon), \quad J=\left(v_{p}-\delta, v_{p}+\delta\right), \quad K=[0, \eta),
$$

with $\epsilon \in(0,1 / 2), \delta$ in $\left(0, v_{p} / 2\right)$ and $\eta>0$ to be chosen later, such that $\overline{I \times J \times K} \subset U$. Let $\psi_{I}$ be a function in $C_{c}^{\infty}(I)$ such that $\psi_{I}(t)=1$ for $t \in \frac{1}{2} I=(1-\epsilon / 2,1+\epsilon / 2)$. Since

$$
1=\psi_{I} \otimes \mathbf{1}_{J} \otimes \mathbf{1}_{K}+\psi_{I} \otimes \mathbf{1}_{J^{c}} \otimes \mathbf{1}_{K}+\left(1-\psi_{I}\right) \otimes 1 \otimes \mathbf{1}_{K}+1 \otimes 1 \otimes \mathbf{1}_{K^{c}},
$$

we may write

$$
\int_{0}^{1 /(2 \sigma)} t^{-d / 2-1} g(\sigma t) e^{u \Phi(t, v, \sigma)} \mathrm{d} t=\sum_{j=1}^{4} I_{j}(u, v, \sigma),
$$

where

$$
\begin{aligned}
& I_{1}(u, v, \sigma)=\mathbf{1}_{J}(v) \mathbf{1}_{K}(\sigma) \int_{0}^{\infty} \psi_{I}(t) t^{-d / 2-1} g(\sigma t) e^{u \Phi(t, v, \sigma)} \mathrm{d} t \\
& I_{2}(u, v, \sigma)=\mathbf{1}_{J^{c}}(v) \mathbf{1}_{K}(\sigma) \int_{0}^{\infty} \psi_{I}(t) t^{-d / 2-1} g(\sigma t) e^{u \Phi(t, v, \sigma)} \mathrm{d} t \\
& I_{3}(u, v, \sigma)=\mathbf{1}_{K}(\sigma) \int_{0}^{1 /(2 \sigma)}\left(1-\psi_{I}(t)\right) t^{-d / 2-1} g(\sigma t) e^{u \Phi(t, v, \sigma)} \mathrm{d} t \\
& I_{4}(u, v, \sigma)=\mathbf{1}_{K^{c}}(\sigma) \int_{0}^{1 /(2 \sigma)} t^{-d / 2-1} g(\sigma t) e^{u \Phi(t, v, \sigma)} \mathrm{d} t .
\end{aligned}
$$

Note that in the first two integrals we may take $\infty$ as the upper limit of integration, because the support of $\psi_{I}$ is contained in $(0,1 /(2 \sigma))$ for all $\sigma<\eta$, provided that $\epsilon$ and $\eta$ are sufficiently small.

CASE (i). To prove the estimate for $0<\sigma<\eta$ we must estimate $I_{j}(u, v, \sigma)$ for $j=1,2,3$. Consider first $I_{1}(u, v, \sigma)$. We shall apply the method of stationary phase described above, with $\Psi(t, v, \sigma)=\psi_{I}(t) t^{-d / 2-1} g(\sigma t)$. Then we may choose $\epsilon, \delta$ and $\eta$ so small that $|t(\nu, \sigma)-1|$ and $|\sigma t(\nu, \sigma)|$ are less than $1 / 2$ and $\left|\Phi_{t t}^{\prime \prime}(t(v, \sigma), v, \sigma)\right| \geq c>0$ for all $(t, v, \sigma)$ in $I \times J \times K$. Indeed, the first two estimates are obvious and the third follows by continuity from the fact 
that $\left|\Phi_{t t}^{\prime \prime}\left(1, v_{p}, 0\right)\right|>0$. Hence the coefficients of $L_{j}$ are analytic functions of $(t, v, \sigma)$ in $I \times J \times K$. Now,

$$
L_{j} \Psi(t, v, \sigma)=\sum_{m=0}^{2 j} a_{m}(t, v, \sigma) \partial_{t}^{m} \psi_{I}(t),
$$

where the functions $a_{m}$ are analytic in all their variables, because they are products of coefficients of $L_{j}$ and derivatives of $t^{-d / 2-1} g(\sigma t)$, of order at most $2 k$. Thus $\left(a_{m}\right)_{0}(\nu, \sigma)=a_{m}(t(\nu, \sigma), \nu, \sigma)$ by (5.14). Since $\psi_{I}(t)$ is constant near the point 1 , it is an easy matter to see that

$$
\left|\left(L_{j} \Psi\right)_{0}(\nu, \sigma)\right| \leq C(\Phi)\|g\|_{C^{2 k}(\mathcal{U})} \quad \forall j=0, \ldots, k .
$$

Thus by (5.12), (5.13) and (5.14)

$$
\left|I_{1}(u, v, \sigma)\right| \leq C_{k}\left[e^{u \operatorname{Re} \Phi(t(v, \sigma), v, \sigma)} u^{-1 / 2}+u^{-k}\right] \mathbf{1}_{J}(v) \mathbf{1}_{K}(\sigma) \quad \forall u \geq 1 .
$$

We claim that it is possible to choose $\delta$ and $\eta$ so small that there exists a constant $c>0$ such that

$$
\operatorname{Re} \Phi(t(v, \sigma), v, \sigma) \leq-c\left(\left(\nu-v_{p}\right)^{2}+\sigma^{2}\right) \quad \forall(\nu, \sigma) \in J \times K .
$$

Assuming this claim for the moment, it follows immediately that

$$
\left|I_{1}(u, v, \sigma)\right| \leq C_{k}\left(u^{-1 / 2} e^{-c u\left(\left(v-v_{p}\right)^{2}+\sigma^{2}\right)}+u^{-k}\right) \mathbf{1}_{J}(v) \mathbf{1}_{K}(\sigma) \quad \forall u \geq 1,
$$

which is the desired estimate for $I_{1}$.

It remains to prove $(5.15)$. We shall first verify that near $\left(v_{p}, 0\right)$

$$
\operatorname{Re} \Phi(t(v, \sigma), v, \sigma) \leq-c\left((\operatorname{Im} t(\nu, \sigma))^{2}+\sigma^{2}\right)
$$

for some $c>0$. Indeed, since $t$ is bounded away from 0, by Lemma 5.3 and Taylor's formula

$$
\begin{aligned}
-c \sigma^{2} & \geq \operatorname{Re} \Phi(t, v, \sigma) \\
& =\operatorname{Re}\left[\Phi(t(v, \sigma), v, \sigma)+\frac{1}{2} \Phi_{t t}^{\prime \prime}(t(v, \sigma), v, \sigma)(t-t(v, \sigma))^{2}+O\left((t-t(v, \sigma))^{3}\right)\right],
\end{aligned}
$$

as $(\nu, \sigma) \rightarrow\left(v_{p}, 0\right)$. By choosing $t=\operatorname{Re} t(\nu, \sigma)$ we obtain that

$\operatorname{Re} \Phi(t(v, \sigma), v, \sigma) \leq-c \sigma^{2}+(\operatorname{Im} t(v, \sigma))^{2}\left[\frac{1}{2} \operatorname{Re} \Phi_{t t}^{\prime \prime}(t(v, \sigma), v, \sigma)+O(\operatorname{Im} t(\nu, \sigma))\right]$ 
and (5.17) follows, since

$$
\lim _{(v, \sigma) \rightarrow\left(v_{p}, 0\right)} \operatorname{Re} \Phi_{t t}^{\prime \prime}(t(v, \sigma), v, \sigma)=\operatorname{Re} \Phi_{t t}^{\prime \prime}\left(1, v_{p}, 0\right)=-\left(\tan \phi_{p}\right)^{-1}<0 .
$$

To conclude the proof of (5.15), we need to compute the Taylor expansion of $t(\nu, \sigma)$ at $\left(v_{p}, 0\right)$. Observe that $\tau$ is an odd function, which implies that $\varphi^{\prime}$ is also odd. Hence $\Phi_{t}^{\prime}(t, v, \sigma)$ is an even function of $\sigma$ and the same is true of $t(\nu, \sigma)$. To find the first order Taylor expansion of $t(\nu, \sigma)$ at $\left(v_{p}, 0\right)$ in powers of $v-v_{p}$ and $\sigma^{2}$, we compute the partial derivatives $\frac{\partial t}{\partial \lambda}\left(1, v_{p}\right)$ and $\frac{\partial t}{\partial \sigma^{2}}\left(1, v_{p}\right)$ of $t(\nu, \sigma)$, by differentiating the identity

$$
\Phi_{t}^{\prime}(t(\nu, \sigma), v, \sigma)=0
$$

with respect to $v$ and $\sigma^{2}$ and evaluating the result at $\left(v_{p}, 0\right)$ by means of $(5.3)$. We obtain that

$$
t(\nu, \sigma)=-i \frac{1}{2 v_{p}^{2}}\left(v-v_{p}\right)+\frac{i}{3 v_{p}} e^{2 i \phi_{p}} \sigma^{2}+O\left(\left(v-v_{p}\right)^{2}+\sigma^{4}\right)
$$

as $(v, \sigma)$ tends to $\left(v_{p}, 0\right)$. Hence,

$$
\operatorname{Im} t(v, \sigma)=-\frac{1}{2 v_{p}^{2}}\left(v-v_{p}\right)+\frac{\cos \left(2 \phi_{p}\right)}{3 v_{p}} \sigma^{2}+O\left(\left(v-v_{p}\right)^{2}+\sigma^{4}\right) .
$$

Therefore,

$$
\begin{aligned}
(\operatorname{Im} t(v, \sigma))^{2}+\sigma^{2} & =\frac{1}{4 v_{p}^{4}}\left(v-v_{p}\right)^{2}+\sigma^{2}+o\left(\left(v-v_{p}\right)^{2}+\sigma^{2}\right) \\
& \geq c\left(\left(v-v_{p}\right)^{2}+\sigma^{2}\right)
\end{aligned}
$$

for some $c>0$ and for all $(v, \sigma) \in J \times K$, provided that $\delta$ and $\eta$ are sufficiently small. The claim (5.15) follows by (5.17). This completes the proof of estimate (5.16). Now we fix $\delta$ so that all the previous assumptions which depend on it hold. For the rest of the proof, $\delta$ shall remain fixed.

Next we estimate $I_{2}(u, v, \sigma)$. Here $|t-1|<\epsilon,\left|v-v_{p}\right|>\delta$ and $0 \leq \sigma<\eta$. We claim that it is possible to choose $\epsilon$ and $\eta$ so small that there exist positive constants $c$ and $C$ such that for all $(t, v, \sigma)$ in $I \times J^{c} \times K$

$$
\begin{aligned}
\operatorname{Re} \Phi(t, v, \sigma) & \leq-c v(t-1)^{2} \\
\left|\Phi_{t}^{\prime}(t, v, \sigma)\right| & \geq c(v+1) \\
\left|\Phi_{t t}^{\prime \prime}(t, v, \sigma)\right| & \leq C(v+1) .
\end{aligned}
$$

Indeed, (5.18) follows from (5.9), because $t<1+\epsilon$. 
To prove (5.19), we only need to observe that by (5.5) and (5.7)

$$
\begin{aligned}
\left|\Phi_{t}^{\prime}(t, v, \sigma)\right| \geq\left|\operatorname{Im} \Phi_{t}^{\prime}(t, v, \sigma)\right| & =\left|-\frac{1}{2 v_{p} t^{2}}\left[2\left(v-v_{p}\right) t+v(t-1)^{2}\right]+O\left(\sigma^{2} t\right)\right| \\
& \geq c(v+1),
\end{aligned}
$$

because $\left|v-v_{p}\right|>\delta$ and we may choose $\epsilon$ and $\eta$ small. The proof of (5.20) uses (5.6) and (5.7) and is straightforward. Thus,

$$
\left|\frac{\partial}{\partial t} \frac{\psi_{I}(t) t^{-d / 2-1} g(\sigma t)}{\Phi_{t}^{\prime}(t, v, \sigma)}\right| \leq C(v+1)^{-1} .
$$

Hence, integrating by parts, we obtain that

$$
\begin{aligned}
\left|I_{2}(u, v, \sigma)\right| & \leq C \mathbf{1}_{J} \otimes \mathbf{1}_{K}(\nu, \sigma) u^{-1} \int_{I}\left|\frac{\partial}{\partial t} \frac{\psi_{I}(t) t^{-d / 2-1} g(\sigma t)}{\Phi_{t}^{\prime}(t, v, \sigma)}\right| e^{u \operatorname{Re} \Phi(t, v, \sigma)} \mathrm{d} t \\
& \leq C \mathbf{1}_{J c} \otimes \mathbf{1}_{K}(\nu, \sigma) u^{-1}(v+1)^{-1} \int_{-\infty}^{\infty} e^{-\operatorname{cuv}(t-1)^{2}} \mathrm{~d} t \\
& \leq C \mathbf{1}_{J} \otimes \mathbf{1}_{K}(\nu, \sigma) u^{-3 / 2} v^{-1} .
\end{aligned}
$$

Now we fix $\epsilon$ so that all the previous assumptions which depend on it hold. For the rest of the proof, $\epsilon$ shall remain fixed.

Next, we estimate $I_{3}(u, v, \sigma)$. Here the cut-off $1-\psi_{I}$ vanishes in $(1-$ $\epsilon / 2,1+\epsilon / 2)$. We consider the integrals over $(0,1-\epsilon / 2)$ and $(1+\epsilon / 2,1 /(2 \sigma))$ separately. To estimate the first integral, we observe that when $t \in(0,1-\epsilon / 2)$ there exist positive constants $c, C$ such that

$$
\begin{aligned}
\operatorname{Re} \Phi(t, v, \sigma) & \leq-c v t^{-1} \\
\left|\Phi_{t}^{\prime}(t, v, \sigma)\right| & \geq c t^{-1}\left(v t^{-1}+1\right) \\
\left|\Phi_{t t}^{\prime \prime}(t, v, \sigma)\right| & \leq C t^{-2}\left(v t^{-1}+1\right) .
\end{aligned}
$$

Indeed, (5.22) follows from (5.9), because $t<1-\epsilon / 2$. To prove (5.23), we observe that by (5.7) and (5.5)

$$
\begin{aligned}
\sqrt{2}\left|\Phi_{t}^{\prime}(t, v, \sigma)\right| & \geq\left|\operatorname{Re} \Phi_{t}^{\prime}(t, v, \sigma)\right|+\left|\operatorname{Im} \Phi_{t}^{\prime}(t, v, \sigma)\right| \\
& \geq v\left(t^{-2}-1\right)+\left|-\frac{v}{2 v_{p}}\left(t^{-2}+1\right)+t^{-1}\right|-O\left(\sigma^{2} t\right) \\
& \geq c t^{-1}\left(v t^{-1}+1\right)-O\left(\sigma^{2} t\right) \\
& \geq c t^{-1}\left(v t^{-1}+1\right),
\end{aligned}
$$

if $\eta$ is sufficiently small. Finally, using (5.6) and (5.7), it is easy to see that

$$
\left|\Phi_{t t}^{\prime \prime}(t, v, \sigma)\right| \leq C t^{-2}\left(v t^{-1}+1\right)+O\left(\sigma^{2}\right)
$$


from which (5.24) follows. Now the inequalities (5.23) and (5.24) imply that

$$
\left|\frac{\partial}{\partial t} \frac{\left(1-\psi_{I}(t)\right) t^{-d / 2-1} g(\sigma t)}{\Phi_{t}^{\prime}(t, v, \sigma)}\right| \leq C t^{-d / 2-1},
$$

for $t \in(0,1-\epsilon / 2), 0 \leq \sigma<\eta$ and $v>0$. Integrating by parts and using (5.22), we obtain that

$$
\left|\int_{0}^{1-\epsilon / 2}\left(1-\psi_{I}(t)\right) t^{-d / 2-1} g(\sigma t) e^{u \Phi(t, v, \sigma)} \mathrm{d} t\right|
$$

is bounded by

$$
u^{-1}\left|\int_{0}^{1-\epsilon / 2} t^{-d / 2-1} e^{-c u v t^{-1}} \mathrm{~d} t\right| \leq C u^{-1}(u v)^{-d / 2} e^{-c u v} .
$$

To estimate the integral over $(1+\epsilon / 2,1 /(2 \sigma))$, we observe that there exist positive constants $c, C$ such that

$$
\begin{aligned}
\operatorname{Re} \Phi(t, v, \sigma) & \leq-c v t \\
\left|\Phi_{t}^{\prime}(t, v, \sigma)\right| & \geq c t^{-1}(v t+1) \\
\left|\Phi_{t t}^{\prime \prime}(t, v, \sigma)\right| & \leq C t^{-2}(v t+1),
\end{aligned}
$$

for all $t \in(1+\epsilon / 2,1 /(2 \sigma)), v>0$ and $\sigma>0$. Indeed, (5.26) follows from (5.9), because $t>1+\epsilon / 2$.

To prove (5.27) we write

$$
\Phi_{t}^{\prime}(t, v, \sigma)=w(v, t)+i \sigma \varphi^{\prime}(\sigma t)
$$

where $w(v, t)=-v\left(1-t^{-2}\right)-i\left(\tan \phi_{p}\right) v\left(1+t^{-2}\right)$, and we observe that there exists a positive constant $a$ such that

$$
\pi<\arg (w(t, v))<\frac{3}{2} \pi-a \quad \forall t>1+\epsilon .
$$

Moreover, by (5.11),

$$
0 \leq \arg (i \sigma \varphi(\sigma t)) \leq \pi / 2 \quad \forall t \in(0,1 /(2 \sigma)) .
$$

Therefore there exists a constant $c>0$ such that

$$
\left|\Phi_{t}^{\prime}(t, v, \sigma)\right| \geq c\left(\left|\sigma \varphi^{\prime}(\sigma t)\right|+|w(t, v)|\right)
$$

Hence (5.27) follows, because $|w(t, v)| \geq c v$ and $\left|\sigma \varphi^{\prime}(\sigma t)\right| \geq c_{p} t^{-1}$ for all $\sigma>0$ and all $t$ in $(0,1 /(2 \sigma))$, by (5.10). 
Finally, estimate (5.28) is straightforward.

Now, the inequalities (5.27) and (5.28) imply that

$$
\left|\frac{\partial}{\partial t} \frac{\left(1-\psi_{I}(t)\right) t^{-d / 2-1} g(\sigma t)}{\Phi_{t}^{\prime}(t, v, \sigma)}\right| \leq C t^{-d / 2},
$$

for $t \in(1+\epsilon / 2,1 /(2 \sigma), 0 \leq \sigma<\eta$ and $v>0$. Integrating by parts and using (5.26), we obtain that

$$
\left|\int_{1+\epsilon / 2}^{1 /(2 \sigma)}\left(1-\psi_{I}(t)\right) t^{-d / 2-1} g(\sigma t) e^{u \Phi(t, v, \sigma)} \mathrm{d} t\right|
$$

is bounded by

$$
\text { (5.29) } \frac{C}{u}\left[(2 \sigma)^{d / 2+1} \frac{|g(1 / 2)|}{2 \sigma\left(\frac{v}{2 \sigma}+1\right)} e^{-c u v /(2 \sigma)}+\int_{1+\epsilon / 2}^{1 /(2 \sigma)} t^{-d / 2} e^{-c u v t} \mathrm{~d} t\right] \leq \frac{C}{u(u \nu)} e^{-c u \nu} \text {. }
$$

Considering large and small values of $u v$ separately, we conclude from (5.25) and (5.29) that

$$
\left|I_{3}(t, v, \sigma)\right| \leq C \mathbf{1}_{K}(\sigma) u^{-1}(u v)^{-1 \vee(d / 2)} e^{-c u v} .
$$

Now we fix $\eta$ so that all the previous assumptions which depend on it hold. The proof of case (i) of the proposition follows from the estimates (5.16), (5.21), (5.30) of $I_{j}(t, v, \sigma)$, for $j=1,2,3$.

It remains to prove cases (ii) and (iii). We prove case (iii) first.

CASE (iii). We claim that if $\sigma>3 / 4$, then there exists a constant $c>0$ such that

$$
\operatorname{Re} \Phi(t, v, \sigma) \leq-c\left(\frac{v}{t}+(v \sigma)^{2 / 3}\right) \forall t \in\left(0, \frac{1}{2 \sigma}\right)
$$

Indeed, since $t<2 / 3$, by (5.9) there exists a constant $c>0$ such that

$$
\begin{aligned}
\operatorname{Re} \Phi(t, v, \sigma) & \leq-c \frac{v}{2 t}-c\left(\frac{v}{t}+\sigma^{2} t^{2}\right) \\
& \leq-c \frac{v}{2 t}-c\left(\frac{v}{2 t}\right)^{2 / 3}\left(\sigma^{2} t^{2}\right)^{1 / 3} \\
& =-c \frac{v}{2 t}-c(v \sigma)^{2 / 3}
\end{aligned}
$$


This proves (5.31). Thus

$$
\begin{aligned}
\left|I_{4}(u, v, \sigma)\right| & \leq \max _{[0,1 / 2]}|g| \int_{0}^{1 /(2 \sigma)} t^{-d / 2-1} e^{u \operatorname{Re} \Phi(t, v, \sigma)} \mathrm{d} t \\
& \leq C e^{-c u(v \sigma)^{2 / 3}} \int_{0}^{1 /(2 \sigma)} t^{-d / 2-1} e^{-c u v / t} \mathrm{~d} t \\
& \leq C e^{-c u(v \sigma)^{2 / 3}}(u \nu)^{-d / 2} \int_{c u v \sigma}^{\infty} s^{d / 2-1} e^{-s} \mathrm{~d} s \\
& \leq C e^{-c u(v \sigma)^{2 / 3}}(u v)^{-d / 2} e^{-c u v \sigma},
\end{aligned}
$$

which is the desired estimate when $\sigma>3 / 4$.

CASE (ii). When $\eta \leq \sigma \leq 3 / 4$, we write

$$
I_{4}(u, v, \sigma)=\left(\int_{0}^{2 / 3}+\int_{2 / 3}^{1 /(2 \sigma)}\right) t^{-d / 2-1} g(\sigma t) e^{u \Phi(t, v, \sigma)} \mathrm{d} t .
$$

Estimating the first integral as in the previous case (with $\sigma=3 / 4$ ), we obtain that

$$
\left|\int_{0}^{2 / 3} t^{-d / 2-1} g(\sigma t) e^{u \Phi(t, v, \sigma)} \mathrm{d} t\right| \leq C(u \nu)^{-d / 2} e^{-c u\left(v+v^{2 / 3}\right)} .
$$

To estimate the integral over $(2 / 3,1 /(2 \sigma))$, we write $\Phi(t, v, \sigma)=H(t, v)+$ $i(\varphi(\sigma t)-\log \sigma)$, with

$$
H(t, v)=-v\left[\left(t^{-1}+t-2\right)+i\left(\tan \phi_{p}\right)\left(t^{-1}-t\right)\right] .
$$

Thus, the absolute value of the integral over $[2 / 3,1 /(2 \sigma)]$ is equal to

$$
\left|\int_{2 / 3}^{1 /(2 \sigma)} t^{-d / 2-1} g(\sigma t) e^{i u \varphi(\sigma t)} \frac{1}{u H_{t}^{\prime}(t, v)} \frac{\partial}{\partial t} e^{u H(t, v)} \mathrm{d} t\right| .
$$

Integrating by parts, we may write this integral as a linear combination of two boundary terms of the form

$$
\frac{t^{-d / 2-1} g(\sigma t) e^{u \Phi(t, v, \sigma)}}{u H_{t}^{\prime}(t, v)}, \quad \text { with } \quad t=\frac{2}{3}, \frac{1}{2 \sigma},
$$

and the integral

$$
\int_{2 / 3}^{1 /(2 \sigma)} \frac{\partial}{\partial t}\left[\frac{t^{-d / 2-1} g(\sigma t) e^{i u \varphi(\sigma t)}}{u H_{t}^{\prime}(t, v)}\right] e^{u H(t, v)} \mathrm{d} t .
$$

By (5.9) we have that for $t$ in $[2 / 3,1 /(2 \sigma)]$ and $\sigma>\eta$

$$
\operatorname{Re} \Phi(t, v, \sigma) \leq-c_{p} t^{2} \sigma^{2} \leq-c
$$


for some $c>0$. Moreover

$$
\left|H_{t}^{\prime}(t, v)\right| \geq\left|\operatorname{Im} H_{t}^{\prime}(t, v)\right| \geq\left(\tan \phi_{p}\right) v
$$

for all $t, v>0$. Hence the absolute value of the boundary terms is bounded by $C e^{-c u} v^{-1}$. Since

$$
\begin{aligned}
\left|\frac{\partial}{\partial t}\left[\frac{t^{-d / 2-1} g(\sigma t) e^{i u \varphi(\sigma t)}}{u H_{t}^{\prime}(t, v)}\right] e^{u H(t, v)}\right| & \leq C v^{-1} e^{-u \operatorname{Re} \Phi(t, v, \sigma)} \\
& \leq C v^{-1} e^{-c u},
\end{aligned}
$$

the same bound holds also for the integral in (5.32). This concludes the proof of the proposition.

We are now ready to prove claim (4.11), which, for the reader's convenience, we state again as a proposition.

PROPOSITION 5.5. Suppose that $1<p<2$. For every $b_{0}>0$ there exists a constant $C$ such that

$$
\sup _{x \in \mathbb{R}^{d}} \int_{G_{x}} k_{-b}^{p, i u}(x, y) e^{q_{p}(x, y)} \mathrm{d} y \leq C e^{\phi_{p}^{*} u} \quad \forall u \geq 1 \quad \forall b \in\left(0, b_{0}\right] .
$$

Proof. We recall that

$$
k_{-b}^{p, i u}(x, y)=(1+|u|)^{1 / 2} e^{\phi_{p}^{*} u} \sigma(x, y)^{-d / 2}\left|I\left(u, \frac{a(x, y)}{u}, \sigma(x, y) ; \tilde{g}_{-b}\right)\right|,
$$

where $a(x, y)=\frac{\cos \phi_{p}}{4}|x+y||x-y|, \sigma(x, y)=|x-y| /|x+y|, \tilde{g}_{-b}(t)=$ $g_{-b}\left(t e^{i \phi_{p}}\right)$ and $g_{-b}$ is as in (4.3). In particular $\tilde{g}_{-b}$ is analytic in the unit disk and $\max _{0 \leq j \leq 2 k} \max _{|w| \leq 1 / 2}\left|\tilde{g}_{-b}^{(j)}(w)\right| \leq C$, uniformly with respect to $b$ in $\left(0, b_{0}\right]$. Thus we may apply Proposition 5.4, obtaining an estimate of the kernel $k_{-b}^{p, i u}$ by a sum of several kernels, supported in subsets of the global region. We shall verify that each of them satisfies the desired estimate.

We begin with the estimates corresponding to case (i) of Proposition 5.4. Let the regions $G^{\eta}$ and $G^{\eta, \delta}$ be defined by

$$
G^{\eta}=\{(x, y) \in G: \sigma(x, y)<\eta\} \quad G^{\eta, \delta}=\left\{(x, y) \in G^{\eta}:\left|\frac{a(x, y)}{u}-v_{p}\right|<\delta\right\} .
$$

Define the kernels $k_{i}: \mathbb{R}^{d} \times \mathbb{R}^{d} \rightarrow \mathbb{R}, i=1,2,3,4$ by

$$
\begin{aligned}
& k_{1}(x, y)=u^{-1} \sigma(x, y)^{-d / 2} a(x, y)^{-(1 \vee d / 2)} e^{-c a(x, y)} \mathbf{1}_{G^{\eta}}(x, y) \\
& k_{2}(x, y)=u^{-1 / 2} \sigma(x, y)^{-d / 2} \exp \left\{-c u\left[\left(\frac{a(x, y)}{u}-v_{p}\right)^{2}+\sigma(x, y)^{2}\right]\right\} \mathbf{1}_{G^{\eta, \delta}}(x, y) \\
& k_{3}(x, y)=u^{-k} \sigma(x, y)^{-d / 2} \mathbf{1}_{G^{\eta, \delta}}(x, y) \\
& k_{4}(x, y)=u^{-1 / 2} \sigma(x, y)^{-d / 2} a(x, y)^{-1} \mathbf{1}_{G^{\eta} \backslash G^{\eta, \delta}}(x, y) .
\end{aligned}
$$


We have that

$$
k_{-b}^{p, i u} \mathbf{1}_{G^{\eta}} \leq C(1+|u|)^{1 / 2} e^{\phi_{p}^{*} u}\left(k_{1}+k_{2}+k_{3}+k_{4}\right) .
$$

Therefore, it suffices to show that for $i=1,2,3,4$

$$
\sup _{x \in \mathbb{R}^{d}} \int_{G_{x}^{\eta}} k_{i}(x, y) e^{q p(x, y)} \mathrm{d} y \leq C u^{-1 / 2} \quad \forall u \geq 1 .
$$

We shall use the following facts, whose proof can be found in [7, proof of Proposition 4.4]:

F1: there exists a positive constant $A$ such that

$$
|x-y| \geq \frac{A}{1+|x|} \quad \forall(x, y) \in G
$$

F2: Let $\pi_{\xi}$ denote the orthogonal projection onto the hyperplane orthogonal to $\xi=x /|x|$. For every $\eta$ in $(0,1 / 2)$ there exist positive constants $B, C_{1}, C_{2}, c$ such that

$$
|x| \geq B, \quad C_{1}|x| \leq|x+y| \leq C_{2}|x| \quad \forall(x, y) \in G^{\eta}
$$

and

$$
q_{p}(x, y) \leq-c \frac{|x|\left|\pi_{\xi}(y)\right|^{2}}{|x-y|} \quad \forall(x, y) \in G^{\eta}
$$

Using these facts and the change of variable $z=|x|(y-x)$, one easily sees that for $d \geq 2$

$$
\begin{aligned}
\sup _{x \in \mathbb{R}^{d}} \int_{\left(G^{\eta}\right)_{x}} k_{1}(x, y) e^{q_{p}(x, y)} \mathrm{d} y & \leq C u^{-1} \sup _{|x| \geq B} \int_{|x-y| \geq \frac{A}{1+|x|}}|x-y|^{-d} e^{-c|x||x-y|} \mathrm{d} y \\
& \leq C u^{-1} \int_{|z| \geq \frac{A B}{1+B}}|z|^{-d} e^{-c|z|} \mathrm{d} z \\
& \leq C u^{-1}
\end{aligned}
$$

which implies the desired estimate. When $d=1$ one can reach the same conclusion with a slightly different estimate.

Next we consider $k_{2}$. We observe that, by F2, there exist two positive constants $D_{1}, D_{2}$ depending on $p$, and such that

$$
|x-y| \leq C_{2} \eta|x|, \quad D_{1} u \leq|x||x-y| \leq D_{2} u \quad \forall(x, y) \in G^{\eta, \delta} .
$$


Thus, using $\mathbf{F 1}$ and $\mathbf{F 2}$, it is easy to see that

$$
\sup _{x \in \mathbb{R}^{d}} \int_{\left(G^{\eta, \delta}\right)_{x}} k_{2}(x, y) e^{q_{p}(x, y)} \mathrm{d} y
$$

is bounded by

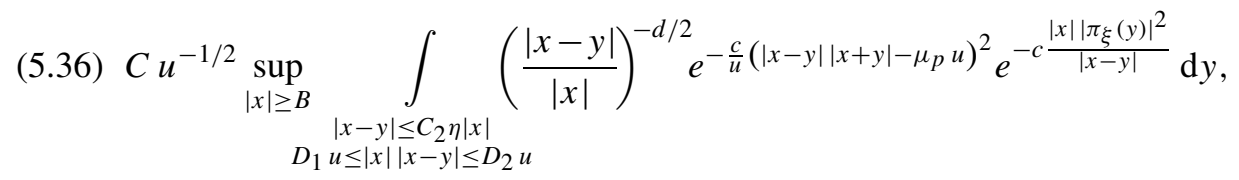
where $\mu_{p}=4 v_{p} / \cos \phi_{p}$. We change variables, letting $y=|x| z+x$. Rotating the $z$ coordinates, we can also assume that the $z_{1}$ axis is parallel to $\xi$. Writing $z=\left(z_{1}, z^{\prime}\right)$, we then have $\left|\pi_{\xi}(y)\right|=|x|\left|\pi_{\xi}(z)\right|=|x|\left|z^{\prime}\right|$. Since $|z| \approx u /|x|^{2}$, it is easily seen that the expression in (5.36) is dominated by

$$
C u^{-(d+1) / 2} \sup _{|x| \geq B}|x|^{2 d} \int_{|z| \leq C_{2} \eta} \exp \left\{-c \frac{|x|^{4}}{u}\left[\left(|2 \xi+z||z|-\mu_{p} \frac{u}{|x|^{2}}\right)^{2}+\left|z^{\prime}\right|^{2}\right]\right\} \mathrm{d} z .
$$

We claim that, if $\eta$ is sufficiently small, there exists a constant $C$ such that

$$
\int_{|z|<C_{2} \eta} \exp \left\{-v\left[(|2 \xi+z||z|-\mu)^{2}+\left|z^{\prime}\right|^{2}\right]\right\} \mathrm{d} z \leq C v^{-d / 2} \quad \forall v>0 \quad \forall \mu \in \mathbb{R} .
$$

This claim implies immediately that

$$
\sup _{x \in \mathbb{R}^{d}} \int_{\left(G^{\eta, \delta}\right)_{x}} k_{2}(x, y) e^{q_{p}(x, y)} \mathrm{d} y \leq C u^{-1 / 2},
$$

which is the desired estimate.

To prove (5.37), we consider the change of variables $\tilde{z}_{1}=|2 \xi+z||z|$, $\tilde{z}_{j}=z_{j}, j=2, \ldots, d$, assuming $|z|<C_{2} \eta$ and $z_{1}>0$. Then

$$
\frac{\partial \tilde{z}_{1}}{\partial z_{1}}=|2 \xi+z| \frac{z_{1}}{|z|}+|z| \frac{\partial|2 \xi+z|}{\partial z_{1}} .
$$

Here $|2 \xi+z|>1$ if $\eta$ is small, and $|\partial| 2 \xi+z\left|/ \partial z_{1}\right| \leq 1$. So for $z_{1} /|z|>2 C_{2} \eta$, we conclude that $\partial \tilde{z}_{1} / \partial z_{1}>C_{2} \eta$. For such $z$, we can thus make the change of variables, and the Jacobian $\partial z / \partial \tilde{z}$ will satisfy $|\partial z / \partial \tilde{z}| \leq\left(C_{2} \eta\right)^{-1}$.

Hence, that part of the integral in (5.37) which corresponds to $z_{1} /|z|>$ $2 C_{2} \eta$ is at most

$$
\left(C_{2} \eta\right)^{-1} \int \exp \left(-v\left(\left(\tilde{z}_{1}-\mu\right)^{2}+\left|\tilde{z}^{\prime}\right|^{2}\right)\right) \mathrm{d} \tilde{z} \leq C v^{-d / 2} .
$$


Considering instead $z_{1} /|z|<-2 C_{2} \eta$, we get the same estimate. Finally, when $\left|z_{1}\right| /|z|<2 C_{2} \eta$, we have $\left|z^{\prime}\right|>c|z|$ for some $c>0$. The corresponding part of the integral in (5.37) is then at most

$$
\int \exp \left(-c^{2} v^{2}|z|^{2}\right) \mathrm{d} z \leq C v^{-d / 2} .
$$

This proves (5.37).

To estimate the contribution of $k_{3}$ we observe that, by arguments similar to those used in the estimate of $k_{2}$, we obtain that

$$
\begin{aligned}
\sup _{x \in \mathbb{R}^{d}} \int_{\left(G^{\eta, \delta}\right)_{x}} k_{3}(x, y) e^{q_{p}(x, y)} \mathrm{d} y & \leq C_{k} u^{-k} \sup _{|x| \geq B}|x|^{d} \int_{D_{1} u /|x|^{2} \leq|z| \leq D_{2} u /|x|^{2}}|z|^{-d / 2} \mathrm{~d} z \\
& \leq C_{k} u^{-k+d / 2} \quad \forall k \in \mathbb{N} .
\end{aligned}
$$

The desired estimate of $k_{3}$ follows, if we choose $k$ sufficiently large.

Next, using F1, F2 and the change of variables $y=|x| z+x$, we obtain that

$$
\sup _{x \in \mathbb{R}^{d}} \int_{\left(G^{\eta}\right)_{x}} k_{4}(x, y) e^{q p(x, y)} \mathrm{d} y,
$$

is dominated by

$$
\begin{aligned}
& C u^{-1 / 2} \sup _{|x| \geq B}|x|^{-2} \int_{\frac{A}{1+|x|} \leq|x-y| \leq C_{2} \eta|x|}\left(\frac{|x-y|}{|x|}\right)^{-(d+2) / 2} e^{-\frac{|x|\left|\pi_{\xi}(y)\right|^{2}}{|x-y|}} \mathrm{d} y \\
& =C u^{-1 / 2} \sup _{|x| \geq B}|x|^{d-2} \int_{\frac{A}{|x|(1+|x|)} \leq|z| \leq C_{2} \eta}|z|^{-(d+2) / 2} e^{-\frac{|x|^{2}\left|\pi_{\xi}(z)\right|^{2}}{|z|}} \mathrm{d} z .
\end{aligned}
$$

Passing to polar coordinates $z=r \omega$, where $r$ is in $\mathbb{R}^{+}$and $|\omega|=1$, we see that (5.38) is equal to

$$
\begin{aligned}
& C u^{-1 / 2} \sup _{|x| \geq B}|x|^{d-2} \int_{\frac{A}{|x|(1+|x|)}}^{C_{2} \eta} r^{(d-4) / 2} \int_{S^{d-1}} e^{-r|x|^{2}\left|\pi_{\xi}(\omega)\right|^{2}} \mathrm{~d} \sigma(\omega) \mathrm{d} r \\
& \leq C u^{-1 / 2} \sup _{|x| \geq B}|x|^{-1} \int_{\frac{A}{|x|(1+|x|)}}^{C_{2} \eta} r^{-3 / 2} \mathrm{~d} r \\
& \leq C u^{-1 / 2}
\end{aligned}
$$

which is the desired estimate.

Next, we pass to the estimate in the region $G_{3 / 4}^{\eta}=\{(x, y) \in G: \eta \leq$ $\sigma(x, y)<3 / 4\}$, which corresponds to case (ii) of Proposition 5.4. Here $k_{-b}^{p, i u}(x, y)$ is dominated by $C(1+|u|)^{1 / 2} e^{\phi_{p}^{*} u}$ times

$$
a(x, y)^{-d / 2} e^{-c u^{1 / 3} a(x, y)^{2 / 3}} e^{-c a(x, y)}+a(x, y)^{-1} e^{-c u}=k_{5}(x, y)+k_{6}(x, y),
$$


say. We observe that $a(x, y) \geq c\left(1+|y|^{2}\right)$ for $(x, y)$ in $G_{3 / 4}^{\eta}$ and $q_{p}(x, y) \leq 0$ for all $(x, y)$. Hence,

$$
\begin{aligned}
\sup _{x \in \mathbb{R}^{d}} \int_{\left(G_{3 / 4}^{\eta}\right) x} k_{5}(x, y) e^{q_{p}(x, y)} \mathrm{d} y & \leq C e^{-c u^{1 / 3}} \sup _{x \in \mathbb{R}^{d}} \int e^{-c|y|^{2}} \mathrm{~d} y \\
& \leq C e^{-c u^{1 / 3}} .
\end{aligned}
$$

Moreover,

$$
\begin{aligned}
\sup _{x \in \mathbb{R}^{d}} \int_{\left(G_{3 / 4}^{\eta}\right) x} k_{6}(x, y) e^{q_{p}(x, y)} \mathrm{d} y & \leq C e^{-c u} \sup _{x \in \mathbb{R}^{d}} \int_{\left(G_{3 / 4}^{\eta}\right) x}\left(1+|y|^{2}\right)^{-1} e^{-c \frac{|x|\left|\pi_{\xi}(y)\right|^{2}}{|x-y|}} \mathrm{d} y \\
& \leq C e^{-c u} \int\left(1+|y|^{2}\right)^{-1} e^{-c|\pi \xi(y)|^{2}} \mathrm{~d} y \\
& \leq C e^{-c u},
\end{aligned}
$$

as is easily seen by computing the last integral in polar coordinates. This proves the desired estimate in $G_{3 / 4}^{\eta}$.

It remains to prove the estimate in the region $G^{3 / 4}=\{(x, y) \in G: 3 / 4 \leq$ $\sigma(x, y)\}$, which corresponds to case (iii) of Proposition 5.4. Here $k_{-b}^{p, i u}(x, y)$ is dominated by $C(1+|u|)^{1 / 2} e^{\phi_{p}^{*} u}$ times

$$
k_{7}(x, y)=(a(x, y) \sigma(x, y))^{-d / 2} e^{-c u^{1 / 3}(a(x, y) \sigma(x, y))^{2 / 3}} e^{-c a(x, y) \sigma(x, y)} .
$$

We observe that $a(x, y) \sigma(x, y)=C_{p}|x-y|^{2}$. Since $|x-y| \geq c>0$ for $(x, y)$ in $G_{3 / 4}$ and $q_{p}(x, y) \leq 0$ for all $(x, y)$, we get

$$
\begin{aligned}
\sup _{x \in \mathbb{R}^{d}} \int_{\left(G^{3 / 4}\right) x} k_{7}(x, y) e^{q_{p}(x, y)} \mathrm{d} y & \leq C e^{-c u^{1 / 3}} \sup _{x \in \mathbb{R}^{d}} \int e^{-c|x-y|^{2}} \mathrm{~d} y \\
& \leq C e^{-c u^{1 / 3}} .
\end{aligned}
$$

This concludes the proof of the proposition.

\section{REFERENCES}

[1] M. Cowling, Harmonic analysis on semigroups, Ann. of Math. 117 (1983), 267-283.

[2] M. Cowling - I. Doust - A. MCIntosh - A. YAGI, Banach space operators with a bounded $H^{\infty}$ functional calculus, J. Aust. Math. Soc. 60 (1996), 51-89.

[3] M. Cowling - S. MedA, Harmonic analysis and ultracontractivity, Trans. Amer. Math. Soc. 340 (1993), 733-752.

[4] E. B. DAvies, "Heat Kernels and Spectral Theory", Cambridge Tract. in Math. 92, Cambridge University Press, Cambridge, 1989. 
[5] J. B. EPPERSON, The hypercontractive approach to exactly bounding an operator with complex Gaussian kernel, J. Funct. Anal. 87 (1989), 1-30.

[6] J. Garcia-Cuerva - G. Mauceri - P. Suögren - J.L. Torrea, Spectral multipliers for the Ornstein-Uhlenbeck semigroup, J. Anal. Math. 78 (1999), 281-305.

[7] J. García-Cuerva - G. Mauceri - S. Meda - P. Sjögren - J. L. Torrea, Functional Calculus for the Ornstein-Uhlenbeck Operator, J. Funct. Anal. 183 (2001), 413-450.

[8] W. HEBISCH - G. MAUCERI - S. MEDA, Holomorphy of spectral multipliers of the OrnsteinUhlenbeck operator, J. Funct. Anal. 210 (2004), 101-124.

[9] L. HÖRMANDER, Estimates for translation invariant operators in $L^{p}$ spaces, Acta Math. 104 (1960), 93-140.

[10] L. HöRmander, "The Analysis of Linear Partial Differential Operators", Vol. 1 Springer Verlag, Berlin, 1983.

[11] S. Meda, A general multiplier theorem, Proc. Amer. Math. Soc. 110 (1990), 639-647.

[12] E. Nelson, The free Markov field, J. Funct. Anal. 12 (1973), 211-227.

[13] E. M. Stein, "Topics in Harmonic Analysis Related to the Littlewood-Paley Theory", Annals of Math. Studies, No. 63, Princeton N. J., 1970.

Dipartimento di Matematica Università di Genova via Dodecaneso 35 16146 Genova, Italy mauceri@dima.unige.it

Dipartimento di Matematica e Applicazioni Università di Milano-Bicocca via Bicocca degli Arcimboldi 8 20126 Milano, Italy stefano.meda@matapp.unimib.it

Department of Mathematics Göteborg University SE-412 96 Göteborg, Sweden peters@math.chalmers.se 